\title{
Improved Delay-Dependent Robust Stability Criteria for a Class of Uncertain Neutral Type Lur'e Systems with Discrete and Distributed Delays
}

\author{
Kaibo Shi, ${ }^{1}$ Hong Zhu, ${ }^{1}$ Shouming Zhong, ${ }^{2,3}$ Yong Zeng, ${ }^{1}$ and Yuping Zhang ${ }^{1}$ \\ ${ }^{1}$ School of Automation Engineering, University of Electronic Science and Technology of China, Chengdu 611731, China \\ ${ }^{2}$ School of Mathematical Sciences, University of Electronic Science and Technology of China, Chengdu 611731, China \\ ${ }^{3}$ Key Laboratory for Neuroinformation of Ministry of Education, University of Electronic Science and Technology of China, \\ Chengdu 611731, China
}

Correspondence should be addressed to Kaibo Shi; skbs111@163.com

Received 8 October 2013; Revised 28 February 2014; Accepted 21 March 2014; Published 29 April 2014

Academic Editor: Jianping Li

Copyright (C) 2014 Kaibo Shi et al. This is an open access article distributed under the Creative Commons Attribution License, which permits unrestricted use, distribution, and reproduction in any medium, provided the original work is properly cited.

\begin{abstract}
This paper is concerned with the problem of delay-dependent robust stability analysis for a class of uncertain neutral type Lur'e systems with mixed time-varying delays. The system has not only time-varying uncertainties and sector-bounded nonlinearity, but also discrete and distributed delays, which has never been discussed in the previous literature. Firstly, by employing one effective mathematical technique, some less conservative delay-dependent stability results are established without employing the bounding technique and the mode transformation approach. Secondly, by constructing an appropriate new type of Lyapunov-Krasovskii functional with triple terms, improved delay-dependent stability criteria in terms of linear matrix inequalities (LMIs) derived in this paper are much brief and valid. Furthermore, both nonlinearities located in finite sector and infinite one have been also fully taken into account. Finally, three numerical examples are presented to illustrate lesser conservatism and the advantage of the proposed main results.
\end{abstract}

\section{Introduction}

Time delay arises naturally in connection with system process and information flow for different part of dynamic systems. Dynamical systems with time delays, also called dynamic systems with hereditary systems, after effect or dead time, equations with deviating argument, have been investigated extensively for the past few decades due to their wide applications in many research fields, covering nuclear reactor, manual control, engineering systems, neural networks, and other scientific areas [1-42]. The main reason is that time-delay phenomenon frequently happens in various engineering systems and is also a key source of instability and oscillation. Hence, the delayed systems have been widely considered by a great quantity of research results in the recent years. To mention a few, stability analysis is carried out in $[1,3,6,7,11,12,15,17,25,32-34,36] ; H_{\infty}$ controllers and $H_{\infty}$ estimation are investigated in [31, 38, 41]; Lur'e dynamical systems are addressed in [4-6, 26-29]; neutral systems with discrete and distributed delays are researched in [32-34]; passivity analysis and reliable fuzzy control are considered, respectively, in $[39,42]$. For the new results on time-delay systems, see the works [3, 21, 29, 34, 38-42] and references cited therein. Therefore, it is of both theoretical and practical importance to study the problem of stability analysis on delayed systems. So far in the literatures, delayed systems can be classified into two types: retarded type and neutral type [1-12]. The retarded type system only depends on the state delay, while the neutral type system not only defines the derivative term of the current state but also explains the derivative term of the past state. And what is more, the delayed stability criteria are also classified into two categories according to their dependence on the size of the delays, namely, delay-independent stability criteria and delay-dependent stability criteria [13-18]. It is well known that delay-dependent stability criteria are less conservative 
than delay-independent ones when the sizes of time-delays are small.

As everyone knows, many nonlinear physical systems can be expressed as a feedback connection of a linear dynamical system and nonlinearity. One of the important classes of nonlinear systems is the Lur'e dynamical system whose nonlinear element satisfies certain sector constraints. Since the notion of absolute stability was introduced by Lure [19], stability analysis for the Lur'e systems has been extensively studied for several decades [20-29]. Recently, various approaches have been proposed by many researchers to obtain robust stability criteria for time-delay Lur'e control systems. Therefore, many methods have been proposed in these results to reduce the conservatism of the stability criteria, such as model transformation method, the bounding technique, free-weighting matrix method, the method of constructing LyapunovKrasovskii functionals, delay decomposition technique, and weighting-matrix decomposition method. By using suitable Lyapunov-Krasovskii functionals and free-weighting matrices, a new delay-dependent robust stability criterion of the class of uncertain mixed neutral and Lur'e systems has been proposed in [21]. Some conditions have been derived by employing Lyapunov-Krasovskii functionals and generalized convex combination in [26]. Sufficient conditions for the robustly asymptotic stability have been achieved by eliminating nonlinearity and removing free-weighting matrices in [29]. In addition, delayed systems often have a spatial extent because of the presence of an amount of parallel pathways of varying axon sizes and lengths. Then, there may exist either a distribution of conduction velocities along these pathways or a distribution of propagation delays over a period of time in some cases, which may cause another type of time delays, namely, distributed time delays in delayed systems. Hence, the stability problem for neutral systems with nonlinearity perturbation and time delay was thus of interest to a great number of researchers. The results in [30] are neutral-delayindependent by a method based on the equivalent equation of zero and the Leibniz-Newton formula in the derivative of the Lyapunov-Krasovskii functionals. In [32], Li and Zhu have obtained the discrete-, neutral-, and distributed-delaydependent stability conditions for uncertain neutral system with discrete and distributed delays by constructing an augmented Lyapunov-Krasovskii functional and using the freeweighting matrices combined with the bounding technique. In [34], by constructing a proper Lyapunov-Krasovskii functional, Chen et al. have derived some new delay-dependent stability criteria for uncertain neutral system with mixed constant delays by making full use of the free-weighting matrices method and without bounding technique bringing much more conservatism used to deal with these triple-integral terms in [33]; however, the research of uncertain neutral system with mixed constant delays has a lot of limitations because of the delay-dependent robust stability criteria for uncertain neutral systems often connected with time-varying delay. Since time delay and parameter uncertainty exist in Lure systems in practice, the robust stability analysis for uncertain Lur'e systems with time delays still requires further consideration. And theoretically, except the traditional freeweighting matrices approach, how to get rid of the rigorous constraint that the time derivatives of time-varying delays must be less than one still needs much more study.

Motivated by the above discussion, in the paper, it is the first attempt to investigate one effective mathematical technique [29] to extend for a class of uncertain neutral type Lur'e systems with mixed time-varying delays which has not only time-varying uncertainties and sector-bounded nonlinearity, but also discrete and distributed delays. The effective mathematical technique is that dividing $\left[h_{L}, h_{U}\right]$ into $\left[h_{L}, h_{L}+h_{L U}(1) / s\right) \cup \cdots \cup\left[h_{L}+h_{L U}(i-\right.$ $\left.1) / s, h_{L}+h_{L U}(i) / s\right) \cup \cdots \cup\left[h_{L}+h_{L U}(s-1) / s, h_{U}\right]$ is defined as a delay segmentation technique and supposing $h_{L}+h_{L U}(i-1) / s \leq h(t) \leq h_{L}+h_{L U} i / s(i=1,2, \ldots, s$, $\left.h_{L U}=h_{U}-h_{L}\right)$. Based on the mathematical technique, the derivative of $\int_{t-h_{U}}^{t}\left(s-\left(t-h_{U}\right)\right) \dot{x}^{T}(s) Q_{2} \dot{x}(s) d s$ was estimated by $h_{U} \dot{x}^{T}(t) Q_{2} \dot{x}(t)-\left(1 / h_{L}\right) \int_{t-h_{L}}^{t} \dot{x}^{T}(s) d s Q_{1} \int_{t-h_{L}}^{t} \dot{x}(s) d s-$ $\left(s / h_{L U}(s-i+1)\right) \int_{t-h_{U}}^{t-h(t)} \dot{x}^{T}(s) d s Q_{2} \int_{t-h_{U}}^{t-h(t)} \dot{x}(s) d s-(s /$ $\left.h_{L U} i\right) \int_{t-h(t)}^{t-h_{L}} \dot{x}^{T}(s) d s Q_{2} \int_{t-h(t)}^{t-h_{L}} \dot{x}(s) d s$, which plays an important role in the improvement of less conservative results. Secondly, by constructing a new type of Lyapunov-Krasovskii functional with triple terms, improved delay-dependent stability criteria in terms of linear matrix inequalities (LMIs) derived in this paper are brief and effective, which can be easily solved by using MATLAB LIM control toolbox [37]. Furthermore, both nonlinearities located in a finite sector and infinite one have been also fully taken into account. Finally, three numerical examples are given to illustrate the less conservatism of the proposed method.

Notation. Notations used in this paper are fairly standard: $R^{n}$ denotes the $n$-dimensional Euclidean space; $R^{n \times m}$ is the set of all $n \times m$ dimensional matrices; $I$ denotes the identity matrix of appropriate dimensions; $T$ stands for matrix transposition; the notation $X>0$ (resp., $X \geq 0$ ), for $X \in R^{n \times n}$, means that the matrix is real symmetric positive definite (resp., positive semidefinite); $\operatorname{diag}\left\{r_{1}, r_{2}, \ldots, r_{n}\right\}$ denotes block diagonal matrix with diagonal elements $r_{i}, i=1,2, \ldots, m$; the symbol $*$ represents the elements below the main diagonal of a symmetric matrix.

\section{Preliminaries}

Consider the following class of uncertain neutral type Lur'e systems with mixed time-varying delays:

$$
\begin{aligned}
& \dot{x}(t)=(A+\Delta A(t)) x(t)+\left(A_{d}+\Delta A_{d}(t)\right) x(t-h(t)) \\
&+(C+\Delta C(t)) \dot{x}(t-\tau(t)) \\
&+(D+\Delta D(t)) \int_{t-r}^{t} x(s) d s+(B+\Delta B(t)) f(\sigma(t)), \\
& \sigma(t)=H^{T} x(t)=\left[h_{1}, h_{2}, \ldots, h_{m}\right]^{T} x(t), \quad t \geq 0, \\
& x(t)=\phi(t), \quad t \in\left[-\max \left\{h_{U}, \tau_{U}, r\right\}, 0\right],
\end{aligned}
$$

where $x(t) \in R^{n}$ is the state vector, $\sigma(t) \in R^{m}$ is the output vector, $A \in R^{n \times n}, A_{d} \in R^{n \times n}, D \in R^{n \times n}, C \in R^{n \times n}, B \in R^{n \times m}$, 
and $H \in R^{n \times m}$ are known matrices, and $f(\sigma(t)) \in R^{m}$ is the nonlinear function in the feedback path, which is denoted as $f$ for simplicity in the sequel. Its form is formulated as

$$
\begin{aligned}
f(\sigma(t)) & =\left[\begin{array}{lllll}
f_{1}\left(\sigma_{1}(t)\right) & f_{2}\left(\sigma_{2}(t)\right) & \cdots & f_{m}\left(\sigma_{m}(t)\right)
\end{array}\right]^{T} \\
\sigma(t) & =\left[\begin{array}{llll}
\sigma_{1}(t) & \sigma_{2}(t) & \cdots & \sigma_{m}(t)
\end{array}\right]^{T} \\
& =H^{T} x(t)=\left[\begin{array}{llll}
h_{1} & h_{2} & \cdots & h_{m}
\end{array}\right]^{T} x(t),
\end{aligned}
$$

wherein each term $f_{i}\left(\sigma_{i}(t)\right), i=1,2, \ldots, m$, satisfies one of the following sector conditions:

$$
\begin{aligned}
& f_{i}\left(\sigma_{i}(t)\right) \in K_{\left[0, k_{i}\right]} \\
& =\left\{f_{i}\left(\sigma_{i}(t)\right) \mid f_{i}(0)=0,0<\frac{f_{i}\left(\sigma_{i}(t)\right)}{\sigma_{i}(t)} \leq k_{i}, \sigma_{i}(t) \neq 0\right\}
\end{aligned}
$$

or

$$
\begin{aligned}
& f_{i}\left(\sigma_{i}(t)\right) \in K_{[0, \infty]} \\
& =\left\{f_{i}\left(\sigma_{i}(t)\right) \mid f_{i}(0)=0, \frac{f_{i}\left(\sigma_{i}(t)\right)}{\sigma_{i}(t)}>0, \sigma_{i}(t) \neq 0\right\},
\end{aligned}
$$

where $\Delta A(t), \Delta A_{d}(t), \Delta C(t), \Delta D(t)$, and $\Delta B(t)$ are timevarying uncertain matrices of appropriate dimensions, which are assumed to be of the following form:

$$
\begin{gathered}
{\left[\begin{array}{lllll}
\Delta A(t) & \Delta A_{d}(t) & \Delta C(t) & \Delta D(t) & \Delta B(t)
\end{array}\right]} \\
\quad=N F(t)\left[\begin{array}{lllll}
E_{1} & E_{2} & E_{3} & E_{4} & E_{5}
\end{array}\right]
\end{gathered}
$$

where $N, E_{1}, E_{2}, E_{3}, E_{4}$, and $E_{5}$ are known real constant matrices of appropriate dimensions and $F(t)$ is a time-varying uncertain matrix satisfying

$$
F(t)^{T} F(t) \leq I, \quad \forall t \geq 0
$$

By using (5), the uncertain system (1) can be rewritten as follows:

$$
\begin{aligned}
\dot{x}(t)= & (A+\Delta A(t)) x(t)+\left(A_{d}+\Delta A_{d}(t)\right) x(t-h(t)) \\
& +(C+\Delta C(t)) \dot{x}(t-\tau(t)) \\
& +(D+\Delta D) \int_{t-r}^{t} x(s) d s+(B+\Delta B(t)) f(\sigma(t)) \\
= & A x(t)+A_{d} x(t-h(t))+C \dot{x}(t-\tau) \\
& +D \int_{t-r}^{t} x(s) d s+B f(\sigma(t))+N P(t),
\end{aligned}
$$

where

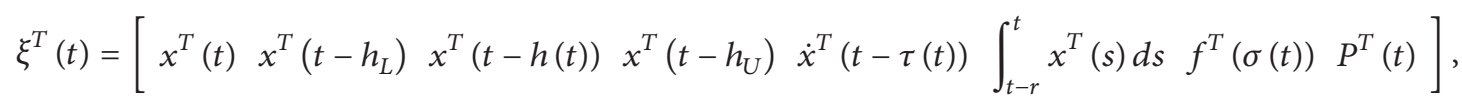

$$
\begin{aligned}
& \xi=\left[\begin{array}{llllllll}
E_{1} & 0 & E_{2} & 0 & E_{3} & E_{4} & E_{5} & 0
\end{array}\right], \quad P(t)=F(t) \xi \xi(t) .
\end{aligned}
$$

The delays $h(t)$ and $\tau(t)$ are time-delaying continuous functions that satisfy

$$
\begin{gathered}
0 \leq h_{L} \leq h(t) \leq h_{U}, \quad \dot{h}(t) \leq h_{D}, \\
0 \leq \tau(t) \leq \tau_{U}, \quad \dot{\tau}(t) \leq \tau_{D}<1,
\end{gathered}
$$

where $h_{L}, h_{U}, h_{D}, \tau_{U}$, and $\tau_{D}$ are known scalars.

The following fact and lemmas are introduced, which will be used in the proof of the main results.

Fact 1 (Schur complement). For a given symmetric matrix $S=S^{T}=\left[\begin{array}{cc}S_{11} & S_{12} \\ * & S_{22}\end{array}\right]$, where $S_{11} \in R^{n \times n}$, the following conditions are equivalent:

(1) $S<0$;

(2) $S_{11}<0, S_{22}-S_{12}^{T} S_{11}^{-1} S_{12}<0$;

(3) $S_{22}<0, S_{11}-S_{12} S_{22}^{-1} S_{12}^{T}<0$.
Lemma 1 (see [35]). For any constant matrix $0<R=R^{T} \epsilon$ $R^{n \times n}$, a scalar $r>0$, a vector function $x:[0, r] \rightarrow R^{n}$ such that the integrations concerned are well defined, and then

$$
-r \int_{0}^{r} x^{T}(s) R x(s) d s \leq-\left(\int_{0}^{r} x(s) d s\right)^{T} R\left(\int_{0}^{r} x(s) d s\right) .
$$

Lemma 2 (see [36]). For any constant matrix $R \in R^{n \times n}, R=$ $R^{T}>0$, a scalar function $h:=h(t)>0$, and a vector-valued function $\dot{x}:[-h, 0] \rightarrow R^{n}$ such that the following integrations are well defined:

$$
\begin{gathered}
-h \int_{t-h}^{t} \dot{x}(s) R \dot{x}(s) d s \leq\left[\begin{array}{c}
x(t) \\
x(t-h)
\end{array}\right]^{T}\left[\begin{array}{cc}
-R & R \\
R & -R
\end{array}\right]\left[\begin{array}{c}
x(t) \\
x(t-h)
\end{array}\right], \\
-\frac{h^{2}}{2} \int_{-h}^{0} \int_{t+\theta}^{t} \dot{x}^{T}(s) R \dot{x}(s) d s d \theta \\
\leq\left[\int_{t-h}^{t} x(s) d s\right]^{T}\left[\begin{array}{cc}
-R & R \\
R & -R
\end{array}\right]\left[\begin{array}{c}
h x(t) \\
\int_{t-h}^{t} x(s) d s
\end{array}\right] .
\end{gathered}
$$




\section{Main Results}

3.1. Stability Analysis in the Finite Sector $K_{\left[0, k_{i}\right]}$. In this section, we will give sufficient conditions under which the system (1) is robustly asymptotically stable.

Theorem 3. For a given positive integer s and some scalars $h_{L}$, $h_{U}, h_{D}, \tau_{U}, \tau_{D}$, and $\epsilon$ with $h_{U}>h_{L}>0, \tau_{D}<1$, and $\epsilon>$ 0 , the system (1) with a nonlinear function $f(\sigma(t))$ satisfying the finite sector condition (3) and time-varying delays $h(t)$ and $\tau(t)$ satisfying (9) is robustly asymptotically stable if there exist $P_{i}^{T}=P_{i}>0(i=1,2, \ldots, 5), Q_{i}^{T}=Q_{i}>0, M_{i}^{T}=M_{i}>$ $0(i=1,2)$, diagonal matrices $R=\operatorname{diag}\left\{r_{1}, r_{2}, \ldots, r_{m}\right\} \geq 0$, and $\Lambda=\operatorname{diag}\left\{\lambda_{1}, \lambda_{2}, \ldots, \lambda_{m}\right\} \geq 0$ with dimensions such that the following symmetric linear matrix inequality holds:

$$
\left[\begin{array}{ccccc}
\Phi+\Pi_{i} & \Gamma^{T} P_{5} & h_{L} \Gamma^{T} Q_{1} & \Gamma^{T} Q_{2} & \frac{r^{2}}{2} \Gamma^{T} M_{2} \\
* & -P_{5} & 0 & 0 & 0 \\
* & * & -Q_{1} & 0 & 0 \\
* & * & * & -\frac{1}{h_{U}} Q_{2} & 0 \\
* & * & * & * & -M_{2}
\end{array}\right]<0
$$

$$
(i=1,2, \ldots, s) \text {, }
$$

where

$$
\begin{aligned}
& \Phi=\left[\begin{array}{cccccccc}
\Phi_{11} & \Phi_{12} & \Phi_{13} & \Phi_{14} & \Phi_{15} & \Phi_{16} & \Phi_{17} & \Phi_{18} \\
* & \Phi_{22} & \Phi_{23} & \Phi_{24} & \Phi_{25} & \Phi_{26} & \Phi_{27} & \Phi_{28} \\
* & * & \Phi_{33} & \Phi_{34} & \Phi_{35} & \Phi_{36} & \Phi_{37} & \Phi_{38} \\
* & * & * & \Phi_{44} & \Phi_{45} & \Phi_{46} & \Phi_{47} & \Phi_{48} \\
* & * & * & * & \Phi_{55} & \Phi_{56} & \Phi_{57} & \Phi_{58} \\
* & * & * & * & * & \Phi_{66} & \Phi_{67} & \Phi_{68} \\
* & * & * & * & * & * & \Phi_{77} & \Phi_{78} \\
* & * & * & * & * & * & * & \Phi_{88}
\end{array}\right], \\
& \Pi_{i}=\left[\begin{array}{cccccccc}
0 & 0 & 0 & 0 & 0 & 0 & 0 & 0 \\
* & \phi_{i 1} & \phi_{i 2} & 0 & 0 & 0 & 0 & 0 \\
* & * & \phi_{i 3} & \phi_{i 4} & 0 & 0 & 0 & 0 \\
* & * & * & \phi_{i 5} & 0 & 0 & 0 & 0 \\
* & * & * & * & 0 & 0 & 0 & 0 \\
* & * & * & * & * & 0 & 0 & 0 \\
* & * & * & * & * & * & 0 & 0 \\
* & * & * & * & * & * & * & 0
\end{array}\right] \\
& \Phi_{11}=P_{1} A+A^{T} P_{1}+P_{2}+P_{3}+P_{4}-Q_{1}-\frac{1}{h_{L}} Q_{2} \\
& +r M_{1}-r^{2} M_{2}+\epsilon E_{1}^{T} E_{1} \\
& \Phi_{12}=Q_{1}+\frac{1}{h_{L}} Q_{2}, \quad \Phi_{13}=P_{1} A_{d}+\epsilon E_{1}^{T} E_{2}, \\
& \Phi_{15}=P_{1} C+\epsilon E_{1}^{T} E_{3}, \quad \Phi_{16}=P_{1} D+r M_{2}+\epsilon E_{1}^{T} E_{4}, \\
& \Phi_{17}=P_{1} B+A^{T} H \Lambda+H K R+\epsilon E_{1}^{T} E_{5}, \\
& \Phi_{18}=P_{1} N, \quad \Phi_{22}=-P_{2}-Q_{1}-\frac{1}{h_{L}} Q_{2},
\end{aligned}
$$

$$
\begin{gathered}
\Phi_{33}=-\left(1-h_{D}\right) P_{3}+\epsilon E_{2}^{T} E_{2}, \quad \Phi_{35}=\epsilon E_{2}^{T} E_{3}, \\
\Phi_{36}=\epsilon E_{2}^{T} E_{4}, \quad \Phi_{37}=A_{d}^{T} H \Lambda+\epsilon E_{2}^{T} E_{5}, \\
\Phi_{44}=-P_{4}, \quad \Phi_{55}=-\left(1-\tau_{D}\right) P_{5}+\epsilon E_{3}^{T} E_{3}, \\
\Phi_{56}=\epsilon E_{3}^{T} E_{4}, \quad \Phi_{57}=C^{T} H \Lambda+\epsilon E_{3}^{T} E_{3}, \\
\Phi_{66}=-\frac{1}{r} M_{1}-M_{2}+\epsilon E_{4}^{T} E_{4}, \quad \Phi_{67}=D^{T} H \Lambda+\epsilon E_{4}^{T} E_{5}, \\
\Phi_{77}=\Lambda H^{T} B+B^{T} H \Lambda-2 R+\epsilon E_{5}^{T} E_{5}, \quad \Phi_{78}=\Lambda H^{T} N, \\
\Phi_{88}=-\epsilon I, \quad \phi_{i 1}=-\frac{s}{h_{L U} i} Q_{2}, \quad \phi_{i 2}=\frac{s}{h_{L U} i} Q_{2}, \\
\phi_{i 4}=\frac{s}{h_{L U}(s-i+1)} Q_{2}, \quad \phi_{i 5}=-\frac{s}{h_{L U}(s-i+1)} Q_{2}, \\
\Gamma=\left[A \quad A_{d}=-\left(\frac{s}{h_{L U} i}+\frac{s \quad D \quad N]}{h_{L U}(s-i+1)},\right.\right. \\
h_{L U}=h_{U}-h_{L}, \text { other terms are } 0 .
\end{gathered}
$$

Proof. Consider a novel augmented Lyapunov-Krasovskii functional for the system (1) as follows:

$$
\begin{aligned}
V\left(x_{t}\right)= & V_{1}\left(x_{t}\right)+V_{2}\left(x_{t}\right)+V_{3}\left(x_{t}\right)+V_{4}\left(x_{t}\right)+V_{5}\left(x_{t}\right) \\
& +V_{6}\left(x_{t}\right)+V_{7}\left(x_{t}\right)+V_{8}\left(x_{t}\right)+V_{9}\left(x_{t}\right)+V_{10}\left(x_{t}\right),
\end{aligned}
$$

where

$$
\begin{aligned}
& V_{1}\left(x_{t}\right)=x^{T}(t) P_{1} x(t), \\
& V_{2}\left(x_{t}\right)=\int_{t-h_{L}}^{t} x^{T}(s) P_{2} x(s) d s, \\
& V_{3}\left(x_{t}\right)=\int_{t-h(t)}^{t} x^{T}(s) P_{3} x(s) d s, \\
& V_{4}\left(x_{t}\right)=\int_{t-h_{U}}^{t} x^{T}(s) P_{4} x(s) d s, \\
& V_{5}\left(x_{t}\right)=\int_{t-\tau(t)}^{t} \dot{x}^{T}(s) P_{5} \dot{x}(s) d s, \\
& V_{6}\left(x_{t}\right)=\int_{t-h_{L}}^{t} h_{L}\left(s-\left(t-h_{L}\right)\right) \dot{x}^{T}(s) Q_{1} \dot{x}(s) d s, \\
& V_{7}\left(x_{t}\right)=\int_{t-h_{U}}^{t}\left(s-\left(t-h_{U}\right)\right) \dot{x}^{T}(s) Q_{2} \dot{x}(s) d s, \\
& V_{8}\left(x_{t}\right)=\int_{t-r}^{t}(s-(t-r)) x^{T}(s) M_{1} x(s) d s, \\
& V_{9}\left(x_{t}\right)=\frac{r^{2}}{2} \int_{-r}^{0} \int_{0}^{\theta} \int_{t+\lambda}^{t} \dot{x}^{T}(s) M_{2} x(s) d s d \lambda d \theta, \\
& V_{10}\left(x_{t}\right)=2 \sum_{i=1}^{N} \lambda_{i} \int_{0}^{\sigma_{i}} f_{i}(s) d s .
\end{aligned}
$$


The derivative of $V\left(x_{t}\right)$ with respect to time along the trajectory (1) is

$$
\begin{aligned}
\dot{V}\left(x_{t}\right)= & \dot{V}_{1}\left(x_{t}\right)+\dot{V}_{2}\left(x_{t}\right)+\dot{V}_{3}\left(x_{t}\right)+\dot{V}_{4}\left(x_{t}\right)+\dot{V}_{5}\left(x_{t}\right) \\
& +\dot{V}_{6}\left(x_{t}\right)+\dot{V}_{7}\left(x_{t}\right)+\dot{V}_{8}\left(x_{t}\right)+\dot{V}_{9}\left(x_{t}\right)+\dot{V}_{10}\left(x_{t}\right) .
\end{aligned}
$$

By utilizing (7), the time derivative of $V_{1}\left(x_{t}\right)$ is obtained as

$$
\begin{aligned}
& \dot{V}_{1}\left(x_{t}\right) \\
& =2 x^{T}(t) P_{1} \dot{x}(t) \\
& =2 x^{T}(t) P_{1}\left[A x(t)+A_{d} x(t-h(t))+C \dot{x}(t-\tau(t))\right. \\
& \left.\quad+D \int_{t-r}^{t} x(s) d s+B f(\sigma(t))+N P(t)\right] \\
& =x^{T}(t)\left(P_{1} A+A^{T} P_{1}\right) x(t)+2 x^{T}(t) P_{1} A_{d} x(t-h(t)) \\
& \quad+2 x^{T}(t) P_{1} C \dot{x}(t-\tau(t))+2 x^{T}(t) P_{1} D \int_{t-r}^{t} x(s) d s \\
& \dot{V}_{2}\left(x_{t}\right)=x^{T}(t) P_{2} x(t)-x^{T}\left(t-h_{L}\right) P_{2} x\left(t-h_{L}\right), \\
& \dot{V}_{3}\left(x_{t}\right) \quad P_{1} B f(\sigma(t))+2 x^{T}(t) P_{1} N P(t), \\
& =x^{T}(t) P_{3} x(t)-(1-\dot{h}(t)) x^{T}(t-h(t)) P_{3} x(t-h(t)) \\
& \leq x^{T}(t) P_{3} x(t)-\left(1-h_{D}\right) x^{T}(t-h(t)) P_{3} x(t-h(t)), \\
& \dot{V}_{4}\left(x_{t}\right)=x^{T}(t) P_{4} x(t)-x^{T}\left(t-h_{U}\right) P_{4} x\left(t-h_{U}\right), \\
& \dot{V}_{5}\left(x_{t}\right) \\
& =\dot{x}^{T}(t) P_{5} \dot{x}(t)-(1-\dot{\tau}(t)) \dot{x}^{T}(t-\tau(t)) P_{5} \dot{x}(t-\tau(t)) \\
& \dot{x}^{T}(t) P_{5} \dot{x}(t)-\left(1-\tau_{D}\right) \dot{x}^{T}(t-\tau(t)) P_{5} \dot{x}(t-\tau(t)) .
\end{aligned}
$$

By utilizing Lemma 2, the time derivative of $V_{6}\left(x_{t}\right)$ is obtained as

$$
\begin{aligned}
\dot{V}_{6}\left(x_{t}\right) \\
=h_{L}^{2} \dot{x}^{T}(t) Q_{1} \dot{x}(t)-\int_{t-h_{L}}^{t} h_{L} \dot{x}^{T}(s) Q_{1} \dot{x}(s) d s \\
\leq h_{L}^{2} \dot{x}^{T}(t) Q_{1} \dot{x}(t)-\left(\int_{t-h_{L}}^{t} \dot{x}(s) d s\right) Q^{T} Q_{1}\left(\int_{t-h_{L}}^{t} \dot{x}(s) d s\right) \\
=h_{L}^{2} \dot{x}^{T}(t) Q_{1} \dot{x}(t)-\left[x(t)-x\left(t-h_{L}\right)\right]^{T} \\
\quad \times Q_{1}\left[x(t)-x\left(t-h_{L}\right)\right] \\
=h_{L}^{2} \dot{x}^{T}(t) Q_{1} \dot{x}(t)-x^{T}(t) Q_{1} x(t)+2 x^{T}(t) Q_{1} x\left(t-h_{L}\right) \\
\quad-x^{T}\left(t-h_{L}\right) Q_{1} x\left(t-h_{L}\right) .
\end{aligned}
$$

By utilizing Lemma 2, for every $i=1,2, \ldots, s$, if $h_{L}+h_{L U}(i-$ $1) / s \leq h(t) \leq h_{L}+h_{L U} i / s, h_{L U}=h_{U}-h_{L}$, the time derivative of $V_{7}\left(x_{t}\right)$ is obtained as

$\dot{V}_{7}\left(x_{t}\right)$

$$
\begin{aligned}
= & h_{U} \dot{x}^{T}(t) Q_{2} \dot{x}(t)-\int_{t-h_{U}}^{t} \dot{x}^{T}(s) Q_{2} \dot{x}(s) d s \\
= & h_{U} \dot{x}^{T}(t) Q_{2} \dot{x}(t)-\int_{t-h_{L}}^{t} \dot{x}^{T}(s) Q_{1} \dot{x}(s) d s \\
& -\int_{t-h(t)}^{t-h_{L}} \dot{x}^{T}(s) Q_{2} \dot{x}(s) d s \\
& -\int_{t-h_{U}}^{t-h(t)} \dot{x}^{T}(s) Q_{2} \dot{x}(s) d s
\end{aligned}
$$$$
\leq h_{U} \dot{x}^{T}(t) Q_{2} \dot{x}(t)-\frac{1}{h_{L}}\left[x(t)-x\left(t-h_{L}\right)\right]^{T}
$$$$
\times Q_{2}\left[x(t)-x\left(t-h_{L}\right)\right]-\frac{s}{h_{L U}(s-i+1)}
$$$$
\times\left[x(t-h(t))-x\left(t-h_{U}\right)\right]^{T}
$$$$
\times Q_{2}\left[x(t-h(t))-x\left(t-h_{U}\right)\right]
$$$$
-\frac{s}{h_{L U} i}\left[x\left(t-h_{L}\right)-x(t-h(t))\right]^{T}
$$$$
\times Q_{2}\left[x\left(t-h_{L}\right)-x(t-h(t))\right]
$$$$
=h_{U} \dot{x}^{T}(t) Q_{2} \dot{x}(t)-\frac{1}{h_{L}} x^{T}(t) Q_{2} x(t)
$$$$
+\frac{2}{h_{L}} x^{T}(t) Q_{2} x\left(t-h_{L}\right)
$$$$
-x^{T}\left(t-h_{L}\right)\left(\frac{1}{h_{L}}+\frac{s}{h_{L U} i}\right) Q_{2} x\left(t-h_{L}\right)
$$$$
+\frac{2 s}{h_{L U} i} x^{T}\left(t-h_{L}\right) Q_{2} x(t-h(t))-x^{T}(t-h(t))
$$$$
\times\left(\frac{s}{h_{L U}(s-i+1)}+\frac{s}{h_{L U} i}\right) Q_{2} x(t-h(t))
$$$$
+\frac{2 s}{h_{L U}(s-i+1)} x^{T}(t-h(t)) Q_{2} x\left(t-h_{U}\right)
$$$$
-\frac{s}{h_{L U}(s-i+1)} x^{T}\left(t-h_{U}\right) Q_{2} x\left(t-h_{U}\right),
$$

$\dot{V}_{8}\left(x_{t}\right)$

$$
=r x^{T}(t) M_{1} x(t)-\int_{t-r}^{t} x^{T}(s) M_{1} x(s) d s
$$

$\leq r x^{T}(t) M_{1} x(t)$

$$
-\frac{1}{r}\left(\int_{t-r}^{t} x(s) d s\right)^{T} M_{1}\left(\int_{t-r}^{t} x(s) d s\right),
$$




$$
\begin{aligned}
& \dot{V}_{9}\left(x_{t}\right)= \frac{r^{4}}{4} \dot{x}^{T}(t) M_{2} \dot{x}(t)-\frac{r^{2}}{2} \int_{-r}^{0} \int_{t+\theta}^{t} \dot{x}^{T}(s) M_{2} \dot{x}(s) d s d \theta \\
& \leq \frac{r^{4}}{4} \dot{x}^{T}(t) M_{2} \dot{x}(t)-r^{2} x^{T}(t) M_{2} x(t) \\
&+2 r x^{T}(t) M_{2} \int_{t-r}^{t} x(s) d s \\
&-\left(\int_{t-r}^{t} x(s) d s\right)^{T} M_{2}\left(\int_{t-r}^{t} x(s) d s\right), \\
& \dot{V}_{10}\left(x_{t}\right) \\
&=2 \sum_{i=1}^{m} \lambda_{i} \dot{\sigma}_{i}(t) f_{i}\left(\sigma_{i}\right)=2 f^{T}(\sigma(t)) \Lambda H^{T} \dot{x}(t) \\
&=2 f^{T}(\sigma(t)) \Lambda H^{T} \\
& \quad \times\left[A x(t)+A_{d} x(t-h(t))+C \dot{x}(t-\tau)\right. \\
&\left.\quad+D \int_{t-r}^{t} x(s) d s+B f(\sigma(t))+N P(t)\right] \\
&+2 x^{T}(t) A^{T} H \Lambda f+2 x^{T}(t-h(t)) A_{d}^{T} H \Lambda f \\
&+2 \dot{x}(t-\tau(t)) C^{T} H \Lambda f+\int_{t-r}^{t} x^{T}(s) d s D^{T} H \Lambda f \\
&+f^{T}\left(\Lambda H^{T} B+B^{T} H \Lambda\right) f+f^{T} \Lambda H^{T} N P(t) .
\end{aligned}
$$

From system (1) with nonlinearity located in the sectors $K_{\left[0, k_{i}\right]}(i=1,2, \ldots, m)$, if there exists $R=\operatorname{diag}\left\{r_{1}, r_{2}, \ldots, r_{m}\right\}$, it is easy to achieve

$$
r_{i} f_{i}\left(\sigma_{i}\right)\left[k_{i} h_{i}^{T} x(t)-f_{i}\left(\sigma_{i}\right)\right] \geq 0, \quad i=1,2, \ldots, m,
$$

which is equivalent to

$$
2\left[x^{T}(t) H K R f(\sigma(t))-f^{T}(\sigma(t)) R f(\sigma(t))\right] \geq 0 ;
$$

also, for any positive scalar $\epsilon$, the following inequality is satisfied:

$$
\epsilon \xi^{T}(t) \xi^{T} \xi \xi(t)-\epsilon P^{T}(t) P(t) \geq 0 .
$$

The combination of (17)-(22) gives that

$$
\begin{array}{r}
\dot{V}\left(x_{t}\right) \\
\leq \xi^{T}(t)\left[\Phi+\Pi_{i}+\Gamma^{T}\left(P_{5}+h_{L}^{2} Q_{1}+h_{U} Q_{2}+\frac{r^{4}}{4} M_{2}\right) \Gamma\right] \xi(t), \\
i=1,2, \ldots, s .
\end{array}
$$

By Fact 1, (23) is equivalent to (12), which implies $\dot{V}\left(x_{t}\right)<$ 0 , which guarantees the robustly asymptotic stability of the system (1). This completes the proof.
When $h_{L}=0$, by simplifying the Lyapunov-Krasovskii functional $V\left(x_{t}\right)$ used in the proof of Theorem 3 to

$$
\begin{aligned}
\bar{V}\left(x_{t}\right)= & V_{1}\left(x_{t}\right)+V_{3}\left(x_{t}\right)+V_{4}\left(x_{t}\right)+V_{5}\left(x_{t}\right)+V_{7}\left(x_{t}\right) \\
& +V_{8}\left(x_{t}\right)+V_{9}\left(x_{t}\right)+V_{10}\left(x_{t}\right) .
\end{aligned}
$$

Theorem 4. For a given positive integer s and some scalars $h_{U}$, $h_{D}, \tau_{U}, \tau_{D}$, and $\epsilon$ with $0<h_{U}, h_{D}<1, \tau_{D}<1$, and $\epsilon>0$, the system (1) with a nonlinear function $f(\sigma(t))$ satisfying the finite sector condition (3) and time-varying delays $h(t)$ and $\tau(t)$ satisfying (9) is robustly asymptotically stable if there exist $P_{i}^{T}=P_{i}>0(i=1,3,4,5), Q_{2}^{T}=Q_{2}>0, M_{i}^{T}=M_{i}>0(i=$ $1,2), R=\operatorname{diag}\left\{r_{1}, r_{2}, \ldots, r_{m}\right\} \geq 0$, and diagonal matrices $\Lambda=\operatorname{diag}\left\{\lambda_{1}, \lambda_{2}, \ldots, \lambda_{m}\right\} \geq 0$ with dimensions such that the following symmetric linear matrix inequality holds:

$$
\left.\begin{array}{cccc}
\Psi+\Lambda_{i} & \Sigma^{T} P_{5} & \Sigma^{T} Q_{2} & \frac{r^{2}}{2} \Sigma^{T} M_{2} \\
* & -P_{5} & 0 & 0 \\
* & * & -\frac{1}{h_{U}} Q_{2} & 0 \\
* & * & * & -M_{2}
\end{array}\right]<0,
$$

where

$$
\begin{aligned}
& \Psi=\left[\begin{array}{ccccccc}
\Psi_{11} & \Psi_{12} & \Psi_{13} & \Psi_{14} & \Psi_{15} & \Psi_{16} & \Psi_{17} \\
* & \Psi_{22} & \Psi_{23} & \Psi_{24} & \Psi_{25} & \Psi_{26} & \Psi_{27} \\
* & * & \Psi_{33} & \Psi_{34} & \Psi_{35} & \Psi_{36} & \Psi_{37} \\
* & * & * & \Psi_{44} & \Psi_{45} & \Psi_{46} & \Psi_{47} \\
* & * & * & * & \Psi_{55} & \Psi_{56} & \Psi_{57} \\
* & * & * & * & * & \Psi_{66} & \Psi_{67} \\
* & * & * & * & * & * & \Psi_{77}
\end{array}\right], \\
& \Lambda_{i}=\left[\begin{array}{ccccccc}
\varphi_{i 1} & \varphi_{i 2} & 0 & 0 & 0 & 0 & 0 \\
* & \varphi_{i 3} & \varphi_{i 4} & 0 & 0 & 0 & 0 \\
* & * & 0 & 0 & 0 & 0 & 0 \\
* & * & * & 0 & 0 & 0 & 0 \\
* & * & * & * & 0 & 0 & 0 \\
* & * & * & * & * & 0 & 0 \\
* & * & * & * & * & * & 0
\end{array}\right], \\
& \Psi_{11}=P_{1} A+A^{T} P_{1}+P_{3}+P_{4}+r M_{1}-r^{2} M_{2}+\epsilon E_{1}^{T} E_{1} \text {, } \\
& \Psi_{12}=P_{1} A_{d}+\epsilon E_{1}^{T} E_{2}, \quad \Psi_{14}=P_{1} C+\epsilon E_{1}^{T} E_{3}, \\
& \Psi_{15}=P_{1} D+r M_{2}+\epsilon E_{1}^{T} E_{4}, \\
& \Psi_{16}=P_{1} B+A^{T} H \Lambda+H K R+\epsilon E_{1}^{T} E_{5}, \\
& \Psi_{17}=P_{1} N, \quad \Psi_{22}=-\left(1-h_{D}\right) P_{3}+\epsilon E_{2}^{T} E_{2}, \\
& \Psi_{24}=\epsilon E_{2}^{T} E_{3}, \quad \Psi_{25}=\epsilon E_{2}^{T} E_{24}, \\
& \Psi_{26}=A_{d} H^{T} \Lambda+\epsilon E_{2}^{T} E_{5}, \quad \Psi_{33}=-P_{4}, \\
& \Psi_{44}=-\left(1-\tau_{D}\right) P_{5}+\epsilon E_{3}^{T} E_{3}, \\
& \Psi_{45}=\epsilon E_{3}^{T} E_{4}, \quad \Psi_{46}=C^{T} H \Lambda+\epsilon E_{3}^{T} E_{5}, \\
& \Psi_{55}=-\frac{1}{r} M_{1}-M_{2}+\epsilon E_{4}^{T} E_{4}, \quad \Psi_{56}=D^{T} H \Lambda+\epsilon E_{4}^{T} E_{5},
\end{aligned}
$$




$$
\begin{aligned}
& \Psi_{66}=\Lambda H^{T} B+B^{T} H \Lambda-2 R+\epsilon E_{5}^{T} E_{5}, \quad \Psi_{67}=\Lambda H^{T} N, \\
& \Psi_{77}=-\epsilon I, \quad \Psi_{i 1}=-\frac{s}{h_{U} i} Q_{2}, \quad \Psi_{i 2}=\frac{s}{h_{U} i} Q_{2}, \\
& \Psi_{i 3}=-\left(\frac{s}{h_{U} i}+\frac{s}{h_{U}(s-i+1)}\right) Q_{2} \text {, } \\
& \Psi_{i 4}=\frac{s}{h_{U}(s-i+1)} Q_{2}, \\
& \Sigma=\left[\begin{array}{lllllll}
A & A_{d} & 0 & C & D & B & N
\end{array}\right], \\
& h_{L U}=h_{U}-h_{L} \text {, other terms are } 0 .
\end{aligned}
$$

When the nonlinear function $f(\sigma(t))$ vanishes, the system (1) reduces to

$$
\begin{gathered}
\dot{x}(t)=(A+\Delta A(t)) x(t)+\left(A_{d}+\Delta A_{d}(t)\right) x(t-h(t)) \\
+(C+\Delta C(t)) \dot{x}(t-\tau(t))+(D+\Delta D) \int_{t-r}^{t} x(s) d s, \\
x(t)=\phi(t), \quad t \in\left[-\max \left\{h_{U}, r\right\}, 0\right] .
\end{gathered}
$$

Similarly, based on Theorem 3, we can obtain the robustly asymptotical stability for system (27) as follows.

Theorem 5. For a given positive integer s and some scalars $h_{L}$, $h_{U}, h_{D}, \tau_{U}, \tau_{D}$, and $\epsilon$ with $h_{U}>h_{L}>0, h_{D}<1, \tau_{D}<1$, and $\epsilon>0$, the system (27) with time-varying delays $h(t)$ and $\tau(t)$ satisfying (9) is robustly asymptotically stable if there exist $P_{i}^{T}=P_{i}>0(i=1,2, \ldots, 5), Q_{i}^{T}=Q_{i}>0$, and $M_{i}^{T}=M_{i}>$ $0(i=1,2)$ with dimensions such that the following symmetric linear matrix inequality holds:

$$
\left[\begin{array}{ccccc}
\Xi+\Delta_{i} & \Upsilon^{T} P_{5} & h_{L} \Upsilon^{T} Q_{1} & \Upsilon^{T} Q_{2} & \frac{r^{2}}{2} \Upsilon^{T} M_{2} \\
* & -P_{5} & 0 & 0 & 0 \\
* & * & -Q_{1} & 0 & 0 \\
* & * & * & -\frac{1}{h_{U}} Q_{2} & 0 \\
* & * & * & * & -M_{2}
\end{array}\right]<0,
$$

where

$$
\Xi=\left[\begin{array}{ccccccc}
\Xi_{11} & \Xi_{12} & \Xi_{13} & \Xi_{14} & \Xi_{15} & \Xi_{16} & \Xi_{17} \\
* & \Xi_{22} & \Xi_{23} & \Xi_{24} & \Xi_{25} & \Xi_{26} & \Xi_{27} \\
* & * & \Xi_{33} & \Xi_{34} & \Xi_{35} & \Xi_{36} & \Xi_{37} \\
* & * & * & \Xi_{44} & \Xi_{45} & \Xi_{46} & \Xi_{47} \\
* & * & * & * & \Xi_{55} & \Xi_{56} & \Xi_{57} \\
* & * & * & * & * & \Xi_{66} & \Xi_{67} \\
* & * & * & * & * & * & \Xi_{77}
\end{array}\right],
$$

$$
\begin{aligned}
& \Delta_{i}=\left[\begin{array}{ccccccc}
0 & 0 & 0 & 0 & 0 & 0 & 0 \\
* & \psi_{i 1} & \psi_{i 2} & 0 & 0 & 0 & 0 \\
* & * & \psi_{i 3} & \psi_{i 4} & 0 & 0 & 0 \\
* & * & * & \phi_{i 5} & 0 & 0 & 0 \\
* & * & * & * & 0 & 0 & 0 \\
* & * & * & * & * & 0 & 0 \\
* & * & * & * & * & * & 0
\end{array}\right], \\
& \Xi_{11}=P_{1} A+A^{T} P_{1}+P_{2}+P_{3}+P_{4}-Q_{1}-\frac{1}{h_{L}} Q_{2} \\
& +r M_{1}-r^{2} M_{2}+\epsilon E_{1}^{T} E_{1} \text {, } \\
& \Xi_{12}=Q_{1}+\frac{1}{h_{L}} Q_{2}, \quad \Xi_{13}=P_{1} A_{d}+\epsilon E_{1}^{T} E_{2}, \\
& \Xi_{15}=P_{1} C+\epsilon E_{1}^{T} E_{3}, \quad \Xi_{16}=P_{1} D+r M_{2}+\epsilon E_{1}^{T} E_{4}, \\
& \Xi_{17}=P_{1} N, \quad \Xi_{22}=-P_{2}-Q_{1}-\frac{1}{h_{L}} Q_{2}, \\
& \Xi_{33}=-\left(1-h_{D}\right) P_{3}+\epsilon E_{2}^{T} E_{2}, \quad \Xi_{35}=\epsilon E_{2}^{T} E_{3}, \\
& \Xi_{36}=\epsilon E_{2}^{T} E_{4}, \quad \Xi_{44}=-P_{4} \text {, } \\
& \Xi_{55}=-\left(1-\tau_{D}\right) P_{5}+\epsilon E_{3}^{T} E_{3} \text {, } \\
& \Xi_{56}=\epsilon E_{3}^{T} E_{4}, \quad \Xi_{66}=-\frac{1}{r} M_{1}-M_{2}+\epsilon E_{4}^{T} E_{4}, \\
& \Xi_{77}=-\epsilon I, \quad \psi_{i 1}=-\frac{s}{h_{L U} i} Q_{2}, \quad \psi_{i 2}=\frac{s}{h_{L U} i} Q_{2}, \\
& \psi_{i 3}=-\left(\frac{s}{h_{L U} i}+\frac{s}{h_{L U}(s-i+1)}\right) Q_{2} \text {, } \\
& \psi_{i 4}=\frac{s}{h_{L U}(s-i+1)} Q_{2} \text {, } \\
& \psi_{i 5}=-\frac{s}{h_{L U}(s-i+1)} Q_{2}, \quad h_{L U}=h_{U}-h_{L}, \\
& \Upsilon=\left[\begin{array}{lllllll}
A & 0 & A_{d} & 0 & C & D & N
\end{array}\right] \text {, other terms are } 0 .
\end{aligned}
$$

\subsection{Stability Analysis in the Infinite Sector $K_{[0, \infty]}$}

Theorem 6. For a given positive integer $s$ and scalars $h_{L}, h_{U}$, $h_{D}, \tau_{U}, \tau_{D}$, and $\epsilon$ with $h_{U}>h_{L}>0, h_{D}<1, \tau_{D}<1$, and $\epsilon>0$, the system (1) with a nonlinear function $f(\sigma(t))$ satisfying the finite sector condition (4) and time-varying delays $h(t)$ and $\tau(t)$ satisfying (9) is robustly asymptotically stable if there exist $P_{i}^{T}=P_{i}>0(i=1,2, \ldots, 5), Q_{i}^{T}=Q_{i}>0$, $M_{i}^{T}=M_{i}>0(i=1,2)$, a scalar $\alpha>0$, and diagonal matrix $\Lambda=\operatorname{diag}\left\{\lambda_{1}, \lambda_{2}, \ldots, \lambda_{m}\right\} \geq 0$ with dimensions such that the following symmetric linear matrix inequality holds:

$$
\left[\begin{array}{ccccc}
\bar{\Phi}+\Pi_{i} & \Gamma^{T} P_{5} & h_{L} \Gamma^{T} Q_{1} & \Gamma^{T} Q_{2} & \frac{r^{2}}{2} \Gamma^{T} M_{2} \\
* & -P_{5} & 0 & 0 & 0 \\
* & * & -Q_{1} & 0 & 0 \\
* & * & * & -\frac{1}{h_{U}} Q_{2} & 0 \\
* & * & * & * & -M_{2}
\end{array}\right],
$$


where

$$
\begin{aligned}
& \bar{\Phi}=\left[\begin{array}{cccccccc}
\Phi_{11} & \Phi_{12} & \Phi_{13} & \Phi_{14} & \Phi_{15} & \Phi_{16} & \bar{\Phi}_{17} & \Phi_{18} \\
* & \Phi_{22} & \Phi_{23} & \Phi_{24} & \Phi_{25} & \Phi_{26} & \Phi_{27} & \Phi_{28} \\
* & * & \Phi_{33} & \Phi_{34} & \Phi_{35} & \Phi_{36} & \Phi_{37} & \Phi_{38} \\
* & * & * & \Phi_{44} & \Phi_{45} & \Phi_{46} & \Phi_{47} & \Phi_{48} \\
* & * & * & * & \Phi_{55} & \Phi_{56} & \Phi_{57} & \Phi_{58} \\
* & * & * & * & * & \Phi_{66} & \Phi_{67} & \Phi_{68} \\
* & * & * & * & * & * & \Phi_{77} & \Phi_{78} \\
* & * & * & * & * & * & * & \Phi_{88}
\end{array}\right], \\
& \bar{\Phi}_{17}=P_{1} B+A^{T} H \Lambda+\alpha H, \quad \bar{\Phi}_{77}=\Lambda H^{T} B+B^{T} H \Lambda .
\end{aligned}
$$

The remaining terms are defined as in Theorem 3.

Proof. For system (1) with nonlinearity located in the sector $K_{[0, \infty]}$, the condition (4) is equivalent to

$$
2 \alpha x^{T}(t) H f(\sigma(t))>0 .
$$

Now, replacing (21) by (32), then combining (17)-(22) and (32) yields

$$
\dot{V}\left(x_{t}\right) \leq \xi^{T}(t) \Psi_{i} \xi(t), \quad i=1,2, \ldots, s .
$$

Then, similarly to the proof of Theorem 3 , the result follows immediately. This completes the proof.

When $h_{L}=0$, we can get a delay-dependent asymptotical stability criterion for the system (1) using the same method of Theorem 4. This result is shown in Theorem 7.

Theorem 7. For a given positive integer $s$ and scalars $h_{U}, h_{D}$, $\tau_{U}, \tau_{D}$, and $\epsilon$ with $0<h_{U}, h_{D}<1, \tau_{D}<1$, and $\epsilon>0$, the system (1) with a nonlinear function $f(\sigma(t))$ satisfying the finite sector condition (4) and time-varying delays $h(t)$ and $\tau(t)$ satisfying $(9)$ is robustly asymptotically stable if there exist $P_{i}^{T}=P_{i}>0(i=1,3,4,5), Q_{2}^{T}=Q_{2}>0, M_{i}^{T}=$ $M_{i}>0(i=1,2)$, a scalar $\alpha>0$, and diagonal matrices $\Lambda=\operatorname{diag}\left\{\lambda_{1}, \lambda_{2}, \ldots, \lambda_{m}\right\} \geq 0$ with dimensions such that the following symmetric linear matrix inequality holds:

$$
\left[\begin{array}{cccc}
\widetilde{\Psi}+\Pi_{i} & \Sigma^{T} P_{5} & \Sigma^{T} Q_{2} & \frac{r^{2}}{2} \Sigma^{T} M_{2} \\
* & -P_{5} & 0 & 0 \\
* & * & -\frac{1}{h_{U}} Q_{2} & 0 \\
* & * & * & -M_{2}
\end{array}\right]<0,
$$

$$
(i=1,2, \ldots, s) \text {, }
$$

where

$$
\begin{gathered}
\widetilde{\Psi}=\left[\begin{array}{ccccccc}
\Psi_{11} & \Psi_{12} & \Psi_{13} & \Psi_{14} & \Psi_{15} & \widetilde{\Psi}_{16} & \Psi_{17} \\
* & \Psi_{22} & \Psi_{23} & \Psi_{24} & \Psi_{25} & \Psi_{26} & \Psi_{27} \\
* & * & \Psi_{33} & \Psi_{34} & \Psi_{35} & \Psi_{36} & \Psi_{37} \\
* & * & * & \Psi_{44} & \Psi_{45} & \Psi_{46} & \Psi_{47} \\
* & * & * & * & \Psi_{55} & \Psi_{56} & \Psi_{57} \\
* & * & * & * & * & \widetilde{\Psi}_{66} & \Psi_{67} \\
* & * & * & * & * & * & \Psi_{77}
\end{array}\right], \\
\widetilde{\Psi}_{16}=P_{1} B+A^{T} H \Lambda+\alpha H, \\
\widetilde{\Psi}_{66}=\Lambda H^{T} B+B^{T} H \Lambda .
\end{gathered}
$$

Remark 8. Recently, Wang et al. [29] present the effective mathematical technique to improve significantly the stability criterion. Motivated by this technique, it is the first attempt of the integral partitioning method to extend to a class of uncertain neutral type Lur'e systems with discrete and distributed delays, which has never been mentioned in the previous literatures.

Remark 9. In the augmented Lyapunov-Krasovskii functional $V\left(x_{t}\right)$, this term $\left(r^{2} / 2\right) \int_{-r}^{0} \int_{0}^{\theta} \int_{t+\lambda}^{t} \dot{x}^{T}(s) M_{2} \dot{x}(s) d s d \lambda d \theta$ plays a key role in reducing the conservatism of our results.

Remark 10. Compared with those in previous methods, this method proposed in this paper has two differences: (i) the item $\int_{t-h_{U}}^{t} \dot{x}^{T}(s) Q_{2} \dot{x}(s) d s$ is added into the Lyapunov functional, which will reduce the conservativeness of results; (ii) the Jensen inequality technique is used, which results in LMIs criteria with less variables than free-weighting matrix approach.

\section{Numerical Examples}

In this section, numerical simulation examples are given to show the effectiveness and correctness of the derived main results.

Example 11. Consider the following class of uncertain neutral type Lur'e systems with mixed time-varying delays:

$$
\begin{aligned}
\dot{x}(t)= & (A+\Delta A(t)) x(t)+\left(A_{d}+\Delta A_{d}(t)\right) x(t-h(t)) \\
& +(C+\Delta C(t)) \dot{x}(t-\tau(t)) \\
& +(D+\Delta D(t)) \int_{t-r}^{t} x(s) d s+(B+\Delta B(t)) f(\sigma(t)),
\end{aligned}
$$

where

$$
\begin{gathered}
A=\left[\begin{array}{cc}
-2 & 0 \\
0 & -1
\end{array}\right], \quad A_{d}=\left[\begin{array}{cc}
-1 & 0 \\
-1 & -1
\end{array}\right], \quad C=\left[\begin{array}{ll}
0 & 0 \\
0 & 0
\end{array}\right], \\
D=\left[\begin{array}{cc}
-0.12 & 0 \\
-0.12 & 0.12
\end{array}\right], \quad B=\left[\begin{array}{l}
-0.3 \\
-0.2
\end{array}\right], \quad H=\left[\begin{array}{l}
0.6 \\
0.8
\end{array}\right], \\
N=\left[\begin{array}{ll}
1.0 & 0.0 \\
0.0 & 1.0
\end{array}\right], \quad E_{1}=\left[\begin{array}{cc}
0.1 & 0 \\
0 & 0.1
\end{array}\right], \\
\left.E_{4}=\left[\begin{array}{cc}
0.1 & 0 \\
0 & 0.1
\end{array}\right], \quad \begin{array}{cc}
0.1 & 0 \\
0 & 0.1
\end{array}\right], \quad E_{3}=\left[\begin{array}{cc}
0.1 & 0 \\
0 & 0.1
\end{array}\right], \\
\Delta A_{d}(t)=\left[\begin{array}{cc}
\alpha_{3} & 0 \\
0 & \alpha_{4}
\end{array}\right], \quad \Delta C(t)=\left[\begin{array}{cc}
0.1 \\
0
\end{array}\right], \quad \Delta A(t)=\left[\begin{array}{cc}
\alpha_{1} & 0 \\
0 & \alpha_{2}
\end{array}\right], \\
0
\end{gathered}
$$


TABLE 1: Allowable time delay $h_{U}$ for $h_{L}=0.1, h_{D}=0.1$, and $r=1$ and different values of $\tau_{D}$ in Example 11 .

\begin{tabular}{lccccccccc}
\hline$\tau_{D}$ & 0.1 & 0.2 & 0.3 & 0.4 & 0.5 & 0.6 & 0.7 & 0.8 & 0.9 \\
\hline Theorem 6 & 3.3587 & 3.1749 & 3.1258 & 3.0987 & 2.9859 & 2.9525 & 2.9012 & 2.8753 & 2.5537 \\
Theorem 3 & 3.5201 & 3.5016 & 3.4857 & 3.4512 & 3.4185 & 3.3125 & 3.1517 & 2.9513 & 2.8718 \\
\hline
\end{tabular}

TABLE 2: Allowable time delay $h_{U}$ for $h_{L}=0, \tau_{D}=0.1$, and $r=1$ and different values of $h_{D}$ in Example 11 .

\begin{tabular}{lccccccccc}
\hline$h_{D}$ & 0.1 & 0.2 & 0.3 & 0.4 & 0.5 & 0.6 & 0.7 & 0.8 & 0.9 \\
\hline Theorem 7 & 4.4132 & 4.2052 & 4.1231 & 4.0532 & 3.8962 & 3.7132 & 3.4891 & 3.2567 & 3.1032 \\
Theorem 4 & 4.5658 & 4.3562 & 4.2578 & 4.1365 & 4.0596 & 3.9862 & 3.7253 & 3.5329 & 3.3568 \\
\hline
\end{tabular}

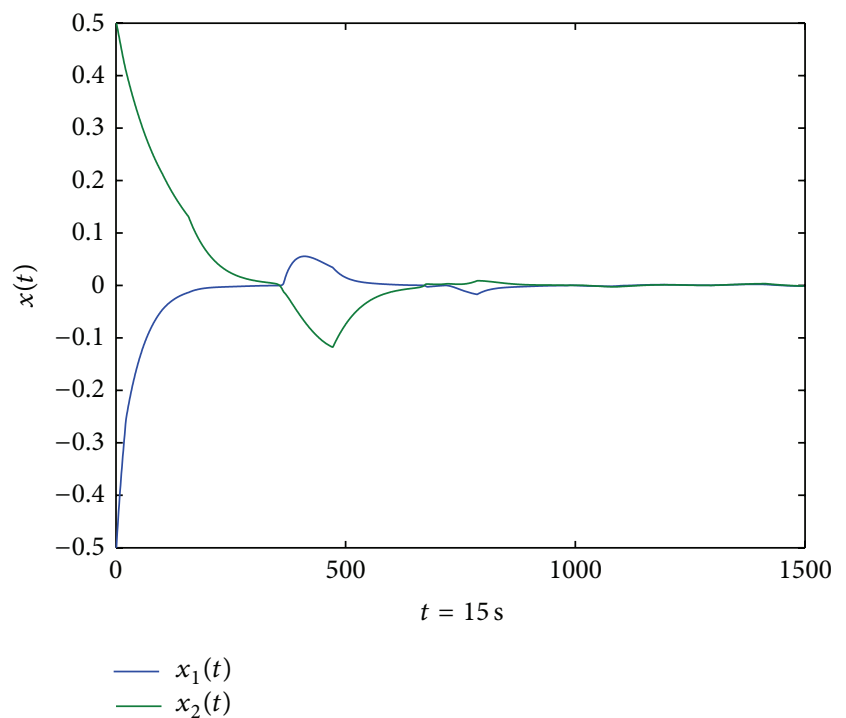

Figure 1: State trajectories of $x(t)$ in the plane $\left(h_{U}=3.5201, h_{L}=\right.$ $0.1, h_{D}=0.1, \tau_{D}=0.1$, and $r=1$, in Example 11, where the initial value is $\left[\begin{array}{ll}-0.5 & 0.5\end{array}\right]^{T}$ ).

$\alpha_{i}(i=1,2, \ldots, 9)$ denote the uncertainties which satisfy

$$
\begin{array}{cc}
-1.6<\alpha_{1}<1.6, & -0.05<\alpha_{2}<0.05, \\
-1.8<\alpha_{3}<1.8, & -0.15<\alpha_{4}<0.15, \\
-1.65<\alpha_{5}<1.65, & -0.25<\alpha_{6}<0.25, \\
-1.5<\alpha_{7}<1.5, & -0.2<\alpha_{8}<0.2, \\
-1.7<\alpha_{9}<1.7 .
\end{array}
$$

Remark 12. For Example 11, our results of the maximum allowable delay bound (MADB) $h_{U}$ for different values of $h_{D}$ and $\tau_{D}$ are listed in Tables 1 and 2. It is clear to show that the maximum allowable delay bound (MADB) $h_{U}$ is dependent on different values of $h_{D}$ and $\tau_{D}$. The values of $h_{D}$ and $\tau_{D}$ increase, respectively, as the maximum allowable delay bound (MADB) $h_{U}$ decreases. In order to verify the stability condition, the simulation results are given as $h_{L}=$ $0.1, h_{D}=0.1, r=1$, and $f(\sigma(t))=\left[f_{1}(\sigma(s))=0.1(\mid s+\right.$ $\left.1|-| s-1 \mid) \quad f_{2}(\sigma(s))=0.1(|s+1|-|s-1|)\right]^{T}$ with initial

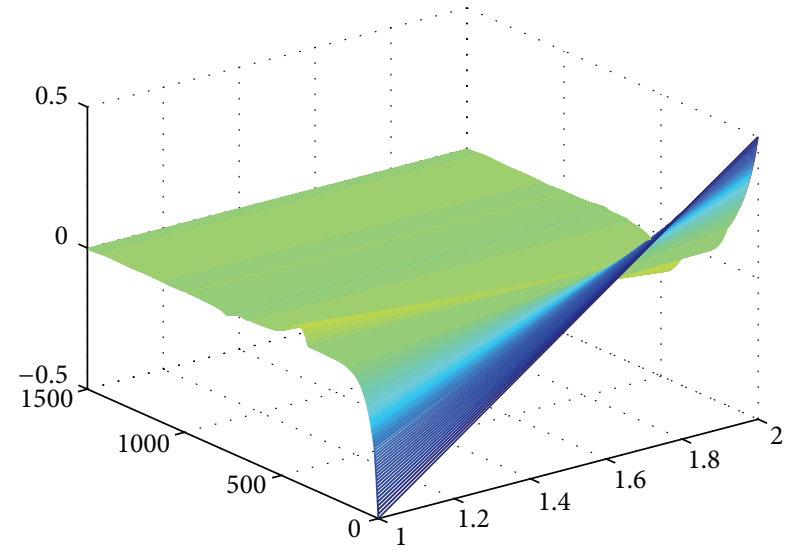

FigURE 2: State trajectories of $x(t)$ on the space $\left(h_{U}=3.5201, h_{L}=\right.$ $0.1, h_{D}=0.1, \tau_{D}=0.1$, and $r=1$, in Example 11, where the initial value is $\left[\begin{array}{ll}-0.5 & 0.5\end{array}\right]^{T}$ ).

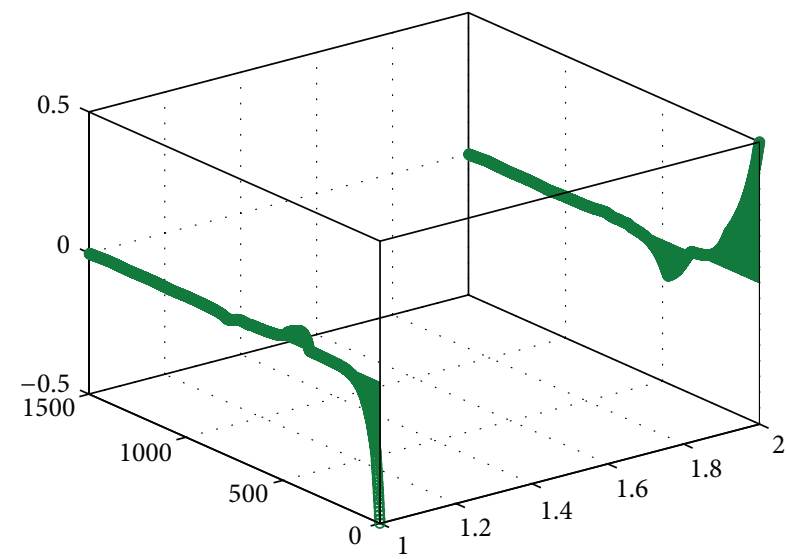

FIGURE 3: State trajectories of $x(t)$ on the space $\left(h_{U}=3.5201, h_{L}=\right.$ $0.1, h_{D}=0.1, \tau_{D}=0.1$, and $r=1$, in Example 11, where the initial value is $\left[\begin{array}{ll}-0.5 & 0.5\end{array}\right]^{T}$ ).

condition $\left[\begin{array}{ll}-0.5 & 0.5\end{array}\right]^{T}$ in Figures 1-4. It is clear that the state trajectories approach to zero asymptotically.

Remark 13. Table 3 shows the maximum values of $h_{U}$ which guarantee stability of this system by applying Theorems 3 and 6 in this paper, where $A_{1}=K[0,0.5), A_{2}=K[0,10), A_{3}=$ $K[0,1000)$, and $A_{4}=K[0, \infty)$. 
TABLE 3: Allowable time delay $h_{U}$ for $h_{L}=0.1, h_{d}=0.1$, and $r=1$ and different values of $\tau_{D}$ in Example 11.

\begin{tabular}{lccccccccc}
\hline$\tau_{D}$ & 0.1 & 0.2 & 0.3 & 0.4 & 0.5 & 0.6 & 0.7 & 0.8 & 0.9 \\
\hline Theorem 3 $\left(f \in A_{1}\right)$ & 3.5127 & 3.4125 & 3.3652 & 3.2158 & 3.1569 & 3.0237 & 2.9853 & 2.9356 & 2.8765 \\
Theorem 3 $\left(f \in A_{2}\right)$ & 3.4419 & 3.3589 & 3.2518 & 3.1321 & 3.0128 & 2.9512 & 2.8758 & 2.8133 & 2.7567 \\
Theorem 3 $\left(f \in A_{3}\right)$ & 3.2158 & 3.1675 & 3.1291 & 3.0962 & 2.9866 & 2.8534 & 2.7962 & 2.7531 & 2.6158 \\
Theorem 6 $\left(f \in A_{4}\right)$ & 3.2013 & 3.1326 & 3.0987 & 2.9765 & 2.9215 & 2.8312 & 2.7538 & 2.7312 & 2.5983 \\
\hline
\end{tabular}

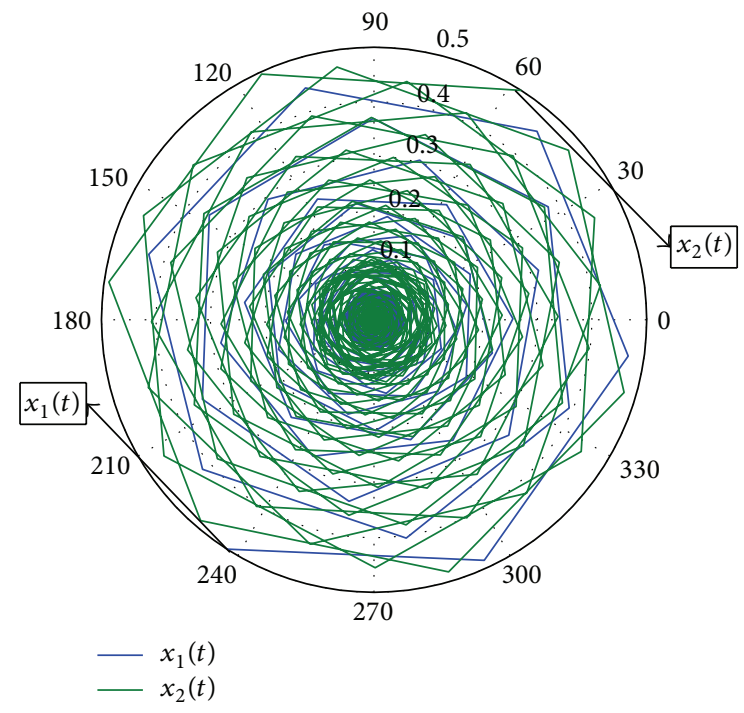

FIGURE 4: State trajectories of $x(t)$ in polar coordinates $\left(h_{U}=3.5201\right.$, $h_{L}=0.1, h_{D}=0.1, \tau_{D}=0.1$, and $r=1$, in Example 11, where the initial value is $\left[\begin{array}{ll}-0.5 & 0.5\end{array}\right]^{T}$ ).

Example 14. Consider the following uncertain system:

$$
\begin{aligned}
\dot{x}(t)= & (A+\Delta A(t)) x(t)+\left(A_{d}+\Delta A_{d}(t)\right) x(t-h) \\
& +(C+\Delta C(t)) \dot{x}(t-\tau)+(D+\Delta D) \int_{t-r}^{t} x(s) d s
\end{aligned}
$$

where

$$
\begin{array}{cc}
A=\left[\begin{array}{cc}
-0.9 & 0.2 \\
0.1 & -0.9
\end{array}\right], & A_{d}=\left[\begin{array}{cc}
-1.1 & -0.2 \\
-0.1 & -1.1
\end{array}\right], \\
C=\left[\begin{array}{cc}
-0.2 & 0 \\
0.2 & -0.1
\end{array}\right], & D=\left[\begin{array}{cc}
-0.12 & 0 \\
-0.12 & 0.12
\end{array}\right], \\
E_{1}=\left[\begin{array}{cc}
0.1 & 0 \\
0 & 0.1
\end{array}\right], & E_{2}=\left[\begin{array}{cc}
0.1 & 0 \\
0 & 0.1
\end{array}\right], \\
E_{3}=\left[\begin{array}{cc}
0.1 & 0 \\
0 & 0.1
\end{array}\right], & E_{4}=\left[\begin{array}{cc}
0.1 & 0 \\
0 & 0.1
\end{array}\right], \\
N=\left[\begin{array}{cc}
1.0 & 0.0 \\
0.0 & 1.0
\end{array}\right], & \Delta A(t)=\left[\begin{array}{cc}
\beta_{1} & 0 \\
0 & \beta_{2}
\end{array}\right], \\
\Delta A_{d}(t)=\left[\begin{array}{cc}
\beta_{3} & 0 \\
0 & \beta_{4}
\end{array}\right], & \Delta C(t)=\left[\begin{array}{cc}
\beta_{5} & 0 \\
0 & \beta_{6}
\end{array}\right], \\
\Delta C(t)=\left[\begin{array}{cc}
\beta_{7} & 0 \\
0 & \beta_{8}
\end{array}\right] .
\end{array}
$$

TABLE 4: Maximum allowable delay bound (MADB) $r$ for $\tau=0.1$ and different values of $h$ in Example 14 .

\begin{tabular}{lcccccc}
\hline$h$ & 0.1 & 0.5 & 1 & 1.5 & 1.6 & 1.7 \\
\hline Li and Zhu [32] & 6.64 & 5.55 & 1.62 & - & - & - \\
Sun et al. [33] & 6.67 & 6.12 & 2.75 & 1.31 & 0.93 & 0.42 \\
Theorem 3.5 in Chen et al. [34] & 6.60 & 4.32 & 0.91 & - & - & - \\
Theorem 3.4 in Chen et al. [34] & 6.67 & 6.02 & 3.19 & 1.50 & 1.25 & 1.02 \\
Theorem 5 $(s=18)$ & 7.32 & 7.13 & 5.89 & 4.56 & 4.08 & 3.16 \\
Theorem 5 $(s=28)$ & 8.16 & 7.96 & 7.68 & 6.58 & 5.32 & 4.65 \\
\hline
\end{tabular}

TABLE 5: Maximum allowable delay bound (MADB) $h$ for $\tau=0.1$ and different values of $r$ in Example 14.

\begin{tabular}{lcccccc}
\hline$r$ & 1 & 2 & 3 & 4 & 5 & 6 \\
\hline Li and Zhu [32] & 1.12 & 0.93 & 0.77 & 0.65 & 0.55 & 0.43 \\
Sun et al. [33] & 1.58 & 1.20 & 0.95 & 0.77 & 0.64 & 0.51 \\
Theorem 3.5 in Chen et al. [34] & 0.97 & 0.77 & 0.63 & 0.52 & 0.44 & 0.35 \\
Theorem 3.4 in Chen et al. [34] & 1.71 & 1.71 & 1.09 & 0.83 & 0.68 & 0.51 \\
Theorem 5 ( $s=18)$ & 2.12 & 2.09 & 1.98 & 1.87 & 1.54 & 1.12 \\
Theorem 5 $(s=28)$ & 2.65 & 2.56 & 2.35 & 2.13 & 1.87 & 1.52 \\
\hline
\end{tabular}

$\beta_{i}(i=1,2, \ldots, 8)$ denote the uncertainties which satisfy

$$
\begin{array}{rlrl}
-1.6 & <\beta_{1}<1.6, & -0.05 & <\beta_{2}<0.05, \\
-1.5 & <\beta_{3}<1.5, & -0.06 & <\beta_{4}<0.06, \\
-1.6 & <\beta_{5}<1.6, & -0.2<\beta_{6}<0.2, \\
-1.4<\beta_{7}<1.4, & -0.1<\beta_{8}<0.1 .
\end{array}
$$

Remark 15. By applying Theorem 5 and solving the LMI (28) using MATLAB LMI control toolbox, we obtain the maximum allowable upper bounds which are listed in Tables 4 and 5. From Tables 4 and 5, it can be seen that our results show significant improvements over the results obtained in $[15,33,34]$. Further, if the values of $s$ become larger, then the upper bound of the delay will also become larger which is the advantage of the delay partitioning approach used in this paper. Figures 5-8 show clearly that the state vector $x(t)$ stabilizes to zero asymptotically.

Example 16. Consider the system (1) with the following parameters [7]:

$$
A=\left[\begin{array}{cc}
-2 & 0.5 \\
0 & -1
\end{array}\right], \quad A_{d}=\left[\begin{array}{cc}
1 & 0.4 \\
0.4 & -1
\end{array}\right]
$$




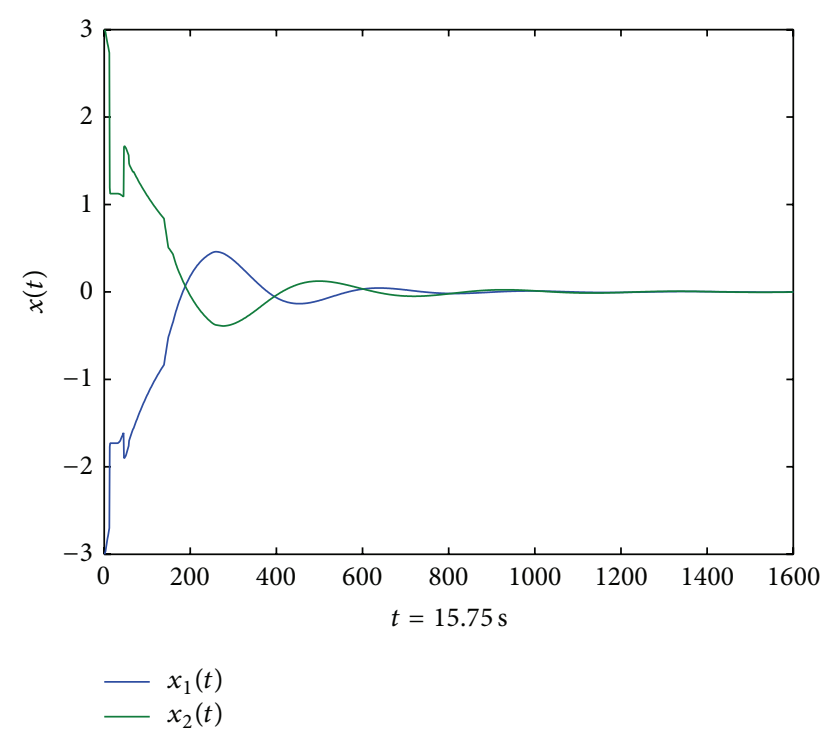

FIGURE 5: State trajectories of $x(t)$ in the plane $(r=8.16, h=0.1$, and $\tau=0.1$ in Example 14, where the initial value is $\left.\left[\begin{array}{ll}-3 & 3\end{array}\right]^{T}\right)$.

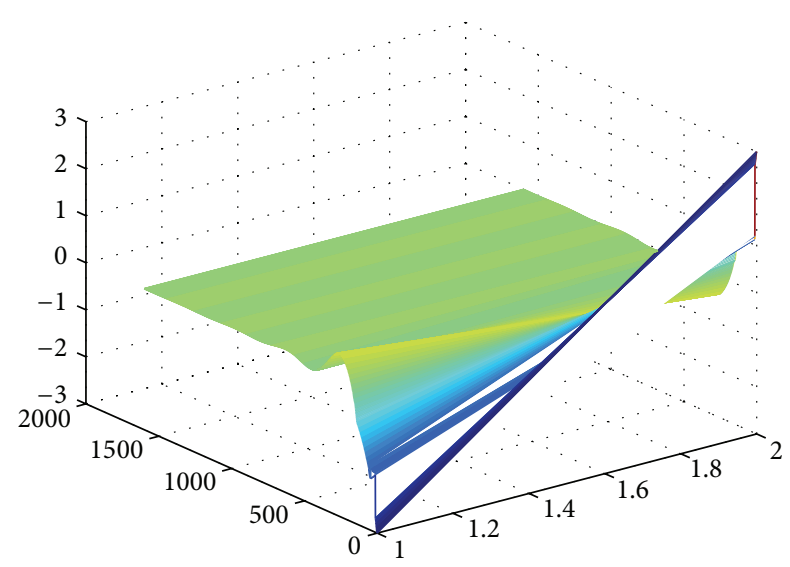

FIGURE 6: State trajectories of $x(t)$ on the space $(r=8.16, h=0.1$, and $\tau=0.1$ in Example 14, where the initial value is $\left.\left[\begin{array}{ll}-3 & 3\end{array}\right]^{T}\right)$.

$$
\begin{gathered}
C=\left[\begin{array}{ll}
0.2 & 0.1 \\
0.1 & 0.2
\end{array}\right], \quad B=\left[\begin{array}{c}
-0.5 \\
-0.75
\end{array}\right], \quad H=\left[\begin{array}{l}
0.2 \\
0.6
\end{array}\right], \\
\Delta A(t)=\Delta A_{d}(t)=\Delta B(t)=\Delta C(t)=\Delta D(t)=D=0 .
\end{gathered}
$$

Remark 17. When $h_{L}=0$, the maximum upper bounds $h_{U}$ of the delay $h(t)$ for different $h_{D}$ and $\tau_{D}$ obtained from [7] and Theorem 3 of this paper are listed in Tables 6, 7, and 8. From the tables, we can see that the proposed result in this paper is less conservative than the one in [7]. Besides, Figures 912 show the simulation results for the state trajectories with initial conditions $\left[\begin{array}{ll}-3 & 3\end{array}\right]^{T}, f(\sigma(t))=\left[f_{1}(\sigma(s))=0.1(\mid s+\right.$ $\left.1|-| s-1 \mid) \quad f_{2}(\sigma(s))=0.1(|s+1|-|s-1|)\right]^{T}$, and time-delay $h_{U}=2.6052$. One can see that the state trajectories approach to zero asymptotically.

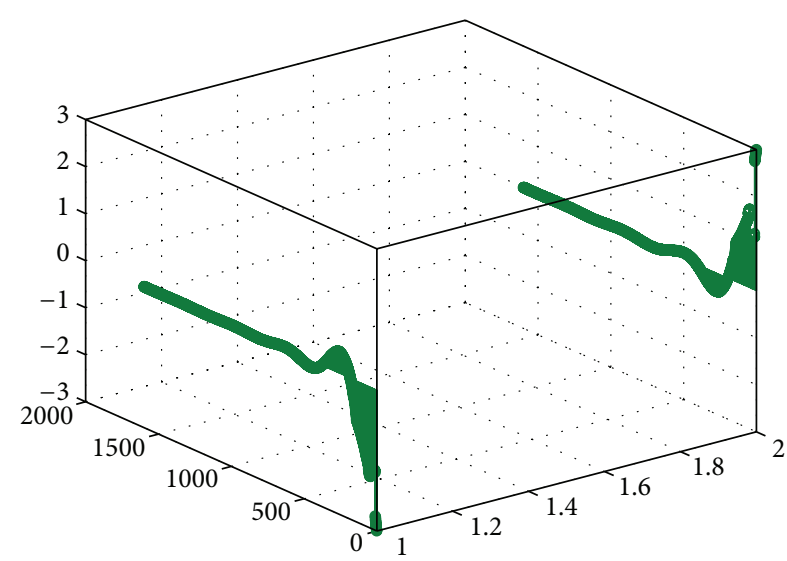

FIgURE 7: State trajectories of $x(t)$ on the space $(r=8.16, h=0.1$, and $\tau=0.1$ in Example 14, where the initial value is $\left.\left[\begin{array}{ll}-3 & 3\end{array}\right]^{T}\right)$.

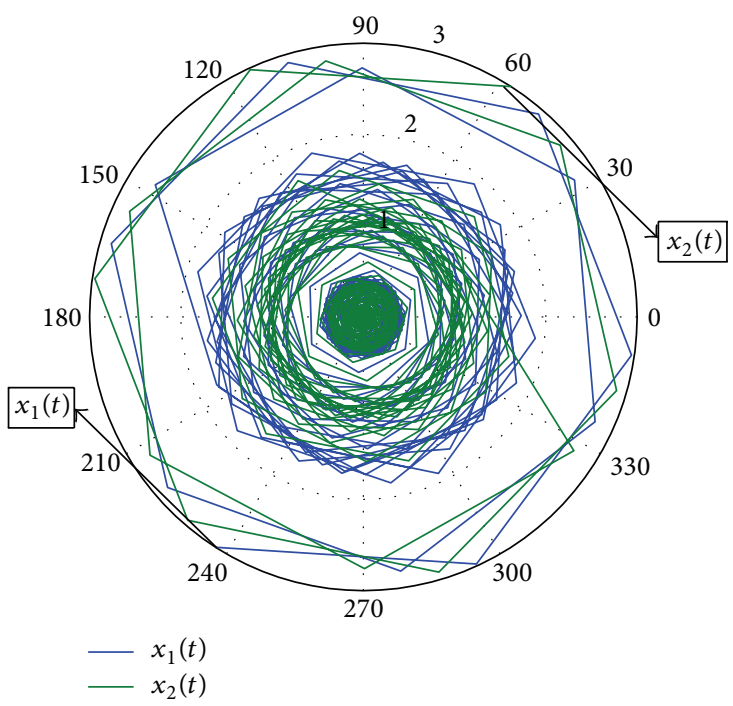

FIGURE 8: State trajectories of $x(t)$ in polar coordinates $(r=8.16, h=$ 0.1 , and $\tau=0.1$ in Example 14, where the initial value is $\left[\begin{array}{ll}-3 & 3\end{array}\right]^{T}$ ).

TABLE 6: Maximum allowable delay bound (MADB) $h_{U}$ for different values of $h_{D}$ in Example 16 .

\begin{tabular}{lcccc}
\hline$h_{D}\left(h_{L}=0, \tau_{D}=0.1\right)$ & 0.2 & 0.4 & 0.6 & 0.8 \\
\hline Gao et al. [7] & 2.5405 & 1.8002 & 1.3605 & 0.7314 \\
Theorem 3 $(s=18)$ & 2.5617 & 1.9216 & 1.5845 & 1.0123 \\
Theorem 3 $(s=28)$ & 2.6052 & 1.9538 & 1.6232 & 1.1659 \\
\hline
\end{tabular}

Finally, in order to better understand the effectiveness of the paper, we give some explanation about Figures 1-12 presented for Examples 11, 14, and 16.

Figures 1, 5, and 9 are 2D line graph using linear axes (vectors create a single line; matrices create one line per column). Plotted variables are

(1) single variable: plot a vector or each column of a matrix as one line versus its index;

(2) $N$ variable pairs: plot each pair of variables in the selected sequence. For example, the sequence varl, 


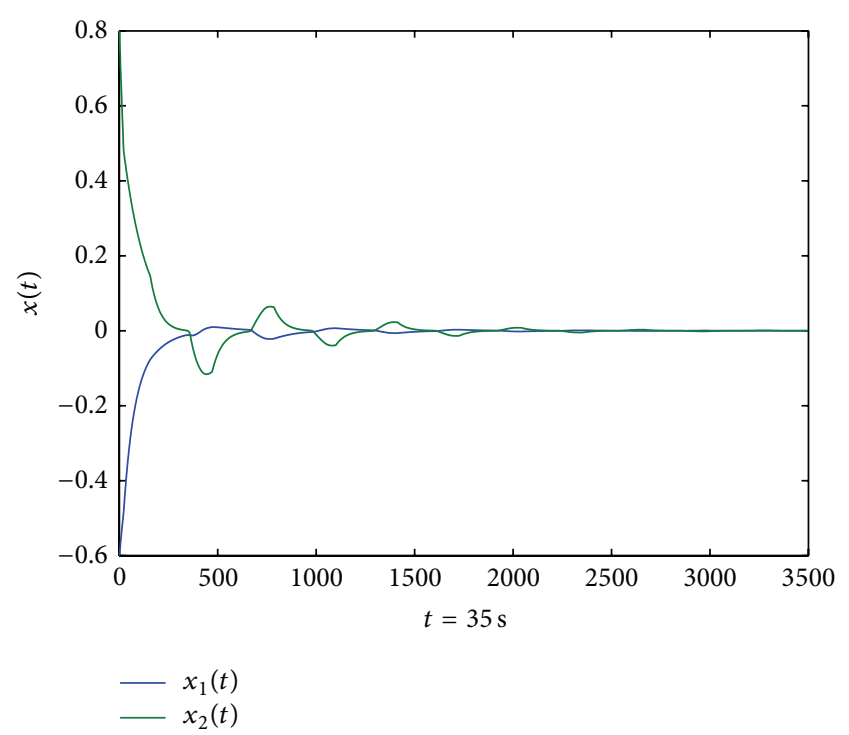

FIGURE 9: State trajectories of $x(t)$ in the plane $\left(h_{U}=2.6052, h_{L}=\right.$ $0, h_{D}=0.2$, and $\tau=0.1$ in Example 16, where the initial value is $\left.\left[\begin{array}{ll}-0.6 & 0.8\end{array}\right]^{T}\right)$.

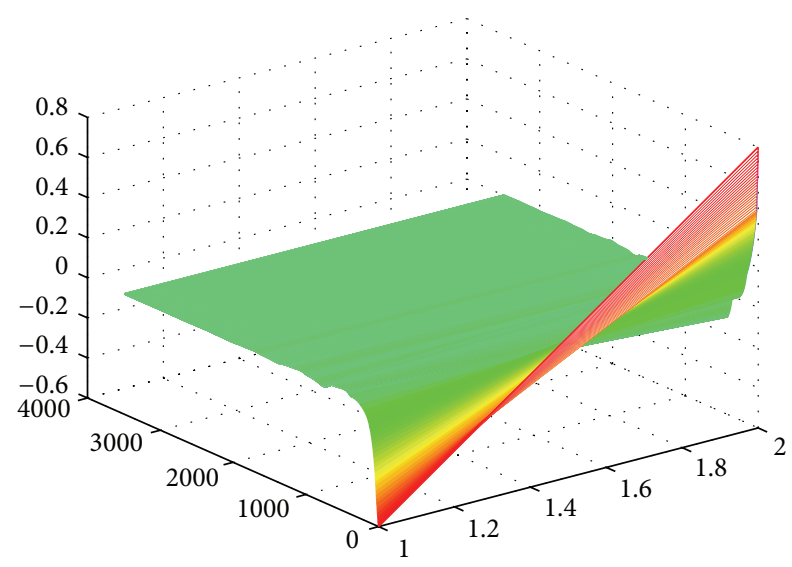

FIGURE 10: State trajectories of $x(t)$ on the space $\left(h_{U}=2.6052, h_{L}=\right.$ $0, h_{D}=0.2$, and $\tau=0.1$ in Example 16, where the initial value is $\left.\left[\begin{array}{ll}-0.6 & 0.8\end{array}\right]^{T}\right)$.

var2, var3, and var4 is plotted as var2 versus var1, var4 versus var3, and so on. Both variables in associated pairs must contain the same number of elements.

Figures 2, 6, and 10 are 3D mesh plot displaying a matrix as a wireframe surface. Plotted variables are as follows:

(1) single variable $(z)$ : plot matrix values as heights above the $x$ - $y$ plane and map data values to colormap;

(2) three variables $(X, Y, Z): X$ and $Y$ are vectors or matrices defining the $x$ and $y$ components of a surface.

Figures 3, 7, and 11 are 3D stem graph (display lines extending from the $x$ - $y$-plane, terminating in circular markers). Plotted variables are as follows:

(1) single variable $(z)$ : when $z$ is a row vector, plot all elements at equally spaced $x$-values against the same

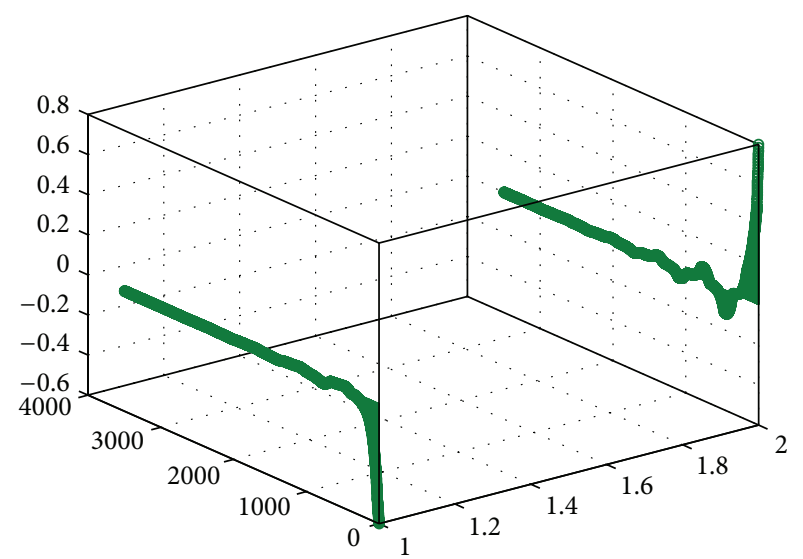

FIGURE 11: State trajectories of $x(t)$ on the space $\left(h_{U}=2.6052, h_{L}=\right.$ $0, h_{D}=0.2$, and $\tau=0.1$ in Example 16, where the initial value is $\left.\left[\begin{array}{ll}-0.6 & 0.8\end{array}\right]^{T}\right)$.

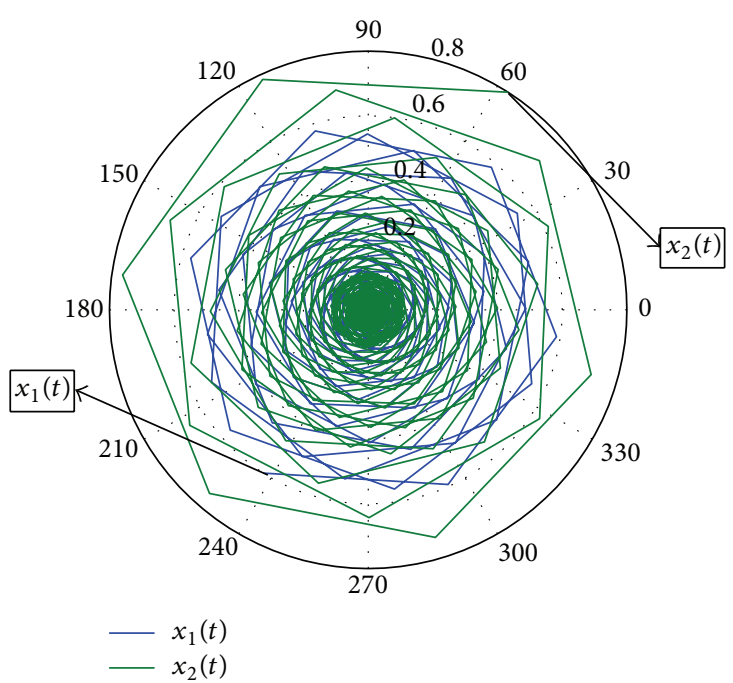

FIGURE 12: State trajectories of $x(t)$ in polar coordinates $\left(h_{U}=\right.$ 2.6052, $h_{L}=0, h_{D}=0.2$, and $\tau=0.1$ in Example 16, where the initial value is $\left[\begin{array}{ll}-0.6 & 0.8\end{array}\right]^{T}$ ).

$y$-value. When $z$ is a column vector, plot all elements at equally spaced $y$-values against the same $x$-value;

(2) three variables $(x, y, z)$ : plot $z$ at values specified by $x$ and $y . x, y$, and $z$ must be of the same size.

Figures 4,8 , and 12 are line graph in polar coordinates. Plotted variables are as follows:

(1) two variables (theta, rho): polar coordinate plot of angle theta (radians) versus radius rho (data units).

Figures 1-12 show clearly that the state trajectories of the system (1), (27) are asymptotically stable from different angles, respectively.

\section{Conclusions}

This paper is focused on the problem of some improved delaydependent robust stability criteria for a class of uncertain 
TABLE 7: Maximum allowable delay bound (MADB) $h_{U}$ for different values of $h_{D}$ in Example 16.

\begin{tabular}{lcccc}
\hline$h_{D}\left(h_{L}=0, \tau_{D}=0.5\right)$ & 0.2 & 0.4 & 0.6 & 0.8 \\
\hline Gao et al. [7] & 2.1066 & 1.5581 & 1.0701 & 0.6329 \\
Theorem 3 $(s=18)$ & 2.1258 & 1.6689 & 1.3210 & 0.9836 \\
Theorem 3 $(s=28)$ & 2.1532 & 1.6986 & 1.3687 & 1.0066 \\
\hline
\end{tabular}

TABLE 8: Maximum allowable delay bound (MADB) $h_{U}$ for different values of $h_{D}$ in Example 16.

\begin{tabular}{lcccc}
\hline$h_{D}\left(h_{L}=0, \tau_{D}=0.9\right)$ & 0.2 & 0.4 & 0.6 & 0.8 \\
\hline Gao et al. [7] & 0.1086 & 0.1022 & 0.0989 & 0.0972 \\
Theorem 3 $(s=18)$ & 0.1219 & 0.1086 & 0.1082 & 0.1082 \\
Theorem 3 $(s=28)$ & 0.1221 & 0.1087 & 0.1083 & 0.1083 \\
\hline
\end{tabular}

neutral type Lure systems with discrete and distributed delays. In the first place, by using one effective mathematical technique, some less conservative delay-dependent stability results are established without employing the bounding technique and the mode transformation approach. In the second place, by constructing an appropriate LyapunovKrasovskii functional with triple terms playing an important role in reducing the conservatism of our results, improved delay-dependent stability criteria in terms of linear matrix inequalities (LMIs) derived in this paper are much simple and valid. What is more, both nonlinearities located in a finite sector and infinite one have been also fully taken into account. Finally, three numerical examples are given to illustrate the less conservatism of the proposed main results.

\section{Conflict of Interests}

The authors declare that there is no conflict of interests regarding the publication of this paper.

\section{Acknowledgment}

This work was supported by National Basic Research Program of China (2010CB732501) and National Natural Science Foundation of China (61273015).

\section{References}

[1] Y. He, M. Wu, J.-H. She, and G.-P. Liu, "Parameter-dependent Lyapunov functional for stability of time-delay systems with polytopic-type uncertainties," IEEE Transactions on Automatic Control, vol. 49, no. 5, pp. 828-832, 2004.

[2] Y. He, Q.-G. Wang, L. Xie, and C. Lin, "Further improvement of free-weighting matrices technique for systems with timevarying delay," IEEE Transactions on Automatic Control, vol. 52, no. 2, pp. 293-299, 2007.

[3] P. Balasubramaniam, R. Krishnasamy, and R. Rakkiyappan, "Delay-dependent stability of neutral systems with timevarying delays using delay-decomposition approach," Applied Mathematical Modelling, vol. 36, no. 5, pp. 2253-2261, 2012.

[4] F. O. Souza, R. M. Palhares, E. M. A. M. Mendes, and L. A. B. Tôrres, "Robust $H_{\infty}$ control for master-slave synchronization of
Lur's systems with time-delay feedback control," International Journal of Bifurcation and Chaos in Applied Sciences and Engineering, vol. 18, no. 4, pp. 1161-1173, 2008.

[5] F. O. Souza, R. M. Palhares, E. M. A. M. Mendes, and L. A. B. Tôrres, "Further results on master-slave synchronization of general Lur's systems with time-varying delay," International Journal of Bifurcation and Chaos in Applied Sciences and Engineering, vol. 18, no. 1, pp. 187-202, 2008.

[6] J. Cao, S. Zhong, and Y. Hu, "Delay-dependent condition for absolute stability of Lurie control systems with multiple time delays and nonlinearities," Journal of Mathematical Analysis and Applications, vol. 338, no. 1, pp. 497-504, 2008.

[7] J. Gao, H. Su, X. Ji, and J. Chu, "Stability analysis for a class of neutral systems with mixed delays and sector-bounded nonlinearity," Nonlinear Analysis: Real World Applications, vol. 9, no. 5, pp. 2350-2360, 2008.

[8] F. Qiu, B. Cui, and Y. Ji, "Delay-dividing approach for absolute stability of Lurie control system with mixed delays," Nonlinear Analysis: Real World Applications, vol. 11, no. 4, pp. 3110-3120, 2010.

[9] F. O. Souza, R. M. Palhares, and V. J. S. Leite, "Improved robust $H_{\infty}$ control for neutral systems via discretised LyapunovKrasovskii functional," International Journal of Control, vol. 81, no. 9, pp. 1462-1474, 2008.

[10] Q.-L. Han, "A new delay-dependent absolute stability criterion for a class of nonlinear neutral systems," Automatica, vol. 44, no. 1, pp. 272-277, 2008.

[11] J. H. Park, "Stability criterion for neutral differential systems with mixed multiple time-varying delay arguments," Mathematics and Computers in Simulation, vol. 59, no. 5, pp. 401-412, 2002.

[12] X.-G. Liu, M. Wu, R. Martin, and M.-L. Tang, "Stability analysis for neutral systems with mixed delays," Journal of Computational and Applied Mathematics, vol. 202, no. 2, pp. 478-497, 2007.

[13] Y. Kuang, Delay Differential Equations With Applications in Population Dynamics, Academic Press, Boston, Mass, USA, 1993.

[14] S. I. Niculescu, Delay Effects on Stability: A Robust Control Approach, Springer, Berlin, Germany, 2001.

[15] J. Cao, S. Zhong, and Y. Hu, "Novel delay-dependent stability conditions for a class of MIMO networked control systems with nonlinear perturbation," Applied Mathematics and Computation, vol. 197, no. 2, pp. 797-809, 2008.

[16] J. K. Hale and S. M. Verduyn Lunel, "Introduction to functional differential equations," in Applied Mathematical Sciences, Springer, New York, NY, USA, 1993.

[17] J. Cao, S. Zhong, and Y. Hu, "Global stability analysis for a class of neural networks with varying delays and control input," Applied Mathematics and Computation, vol. 189, no. 2, pp. 14801490, 2007.

[18] L. Dugard and E. I. Verriest, Stability and Control of Time-Delay Systems, Springer, London, UK, 1998.

[19] A. I. Lur'e, Some Nonlinear Problem in the Theory of Automatic Control, H. M. Stationary Office, London, UK, 1957.

[20] P. Park, "A revisited Popov criterion for nonlinear Lur'e systems with sector-restrictions," International Journal of Control, vol. 68, no. 3, pp. 461-469, 1997.

[21] C. Yin, S.-M. Zhong, and W.-F. Chen, "On delay-dependent robust stability of a class of uncertain mixed neutral and Lur'e dynamical systems with interval time-varying delays," Journal of the Franklin Institute, vol. 347, no. 9, pp. 1623-1642, 2010. 
[22] X. H. Nian, "Delay dependent conditions for absolute stability of Lur's type control systems," Acta Automatica Sinica, vol. 25, no. 4, pp. 564-566, 1999.

[23] B. J. Xu and X. X. Liao, "Criteria for delay-dependent absolute stability of Lurie control systems," Acta Automatica Sinica, vol. 28, no. 2, pp. 317-320, 2002.

[24] D.-Y. Chen and W.-H. Liu, "Delay-dependent robust stability for Lurie control systems with multiple time-delays," Control Theory and Applications, vol. 22, no. 3, pp. 499-502, 2005.

[25] J. Gao, H. Su, X. Ji, and J. Chu, "Stability analysis for a class of neutral systems with mixed delays and sector-bounded nonlinearity," Nonlinear Analysis: Real World Applications, vol. 9, no. 5, pp. 2350-2360, 2008.

[26] T. Li, A. Song, S. Fei, and T. Wang, "Global synchronization in arrays of coupled Lurie systems with both time-delay and hybrid coupling," Communications in Nonlinear Science and Numerical Simulation, vol. 16, no. 1, pp. 10-20, 2011.

[27] J. Cao and S. Zhong, "New delay-dependent condition for absolute stability of Lurie control systems with multiple time-delays and nonlinearities," Applied Mathematics and Computation, vol. 194, no. 1, pp. 250-258, 2007.

[28] J. Tian, S. Zhong, and L. Xiong, "Delay-dependent absolute stability of Lurie control systems with multiple time-delays," Applied Mathematics and Computation, vol. 188, no. 1, pp. 379384, 2007.

[29] Y. Wang, X. Zhang, and Y. He, "Improved delay-dependent robust stability criteria for a class of uncertain mixed neutral and Lur'e dynamical systems with interval time-varying delays and sector-bounded nonlinearity," Nonlinear Analysis: Real World Applications, vol. 13, no. 5, pp. 2188-2194, 2012.

[30] L. Xiong, S. Zhong, and J. Tian, "Novel robust stability criteria of uncertain neutral systems with discrete and distributed delays," Chaos, Solitons \& Fractals, vol. 40, no. 2, pp. 771-777, 2009.

[31] B. Zhang, S. Zhou, and S. Xu, "Delay-dependent $H_{\infty}$ controller design for linear neutral systems with discrete and distributed delays," International Journal of Systems Science, vol. 38, no. 8, pp. 611-621, 2007.

[32] X.-G. Li and X.-J. Zhu, "Stability analysisof neutral systems with distributed delays," Automatica, vol. 44, no. 8, pp. 2197-2201, 2008.

[33] J. Sun, J. Chen, G. P. Liu, and D. Rees, "On robust stability of uncertain neutral systems with discrete and distributed delays," in Proceedings of the American Control Conference (ACC '09), pp. 5469-5473, St. Louis, Mo, USA, June 2009.

[34] H. Chen, Y. Zhang, and Y. Zhao, "Stability analysis for uncertain neutral systems with discrete and distributed delays," Applied Mathematics and Computation, vol. 218, no. 23, pp. 11351-11361, 2012.

[35] K. Gu, "An integral inequaility in the stability problem of timedelay systems," in Proceedings of the 39th IEEE Conference on Decision and Control (CDC '00), pp. 2085-2810, Sydney, Austrlia, 2000.

[36] H. Shao, "Improved delay-dependent globally asymptotic stability criteria for neural networks with a constant delay," IEEE Transactions on Circuits and Systems II, vol. 55, no. 10, pp. 10711075, 2008.

[37] P. Gahinet, A. Nemirovskii, A. J. Labub, and M. Chilall, LMI Control Toolbox, MathWorks, Natick, Mass, USA, 1995.

[38] H. Y. Li, X. J. Jing, and H. R. Karimi, "Output-feedback-based $H_{\infty}$ control for vehicle suspension systems with control delay," IEEE Transactions on Industrial Electronics, vol. 61, no. 1, pp. 436-446, 2014.
[39] H. Li, H. Gao, and P. Shi, "New passivity analysis for neural networks with discrete and distributed delays," IEEE Transactions on Neural Networks, vol. 21, no. 11, pp. 1842-1847, 2010.

[40] H. Gao, T. Chen, and J. Lam, "A new delay system approach to network-based control," Automatica, vol. 44, no. 1, pp. 39-52, 2008.

[41] H. Gao and T. Chen, " $H_{\infty}$ estimation for uncertain systems with limited communication capacity," IEEE Transactions on Automatic Control, vol. 52, no. 11, pp. 2070-2084, 2007.

[42] H. Li, H. Liu, H. Gao, and P. Shi, "Reliable fuzzy control for active suspension systems with actuator delay and fault," IEEE Transactions on Fuzzy Systems, vol. 20, no. 2, pp. 342-357, 2012. 


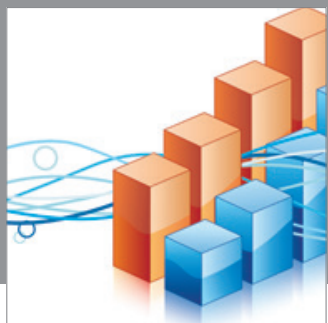

Advances in

Operations Research

mansans

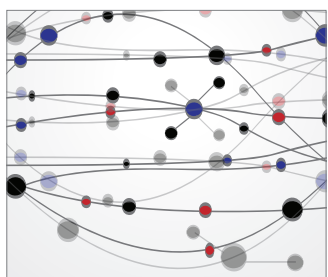

The Scientific World Journal
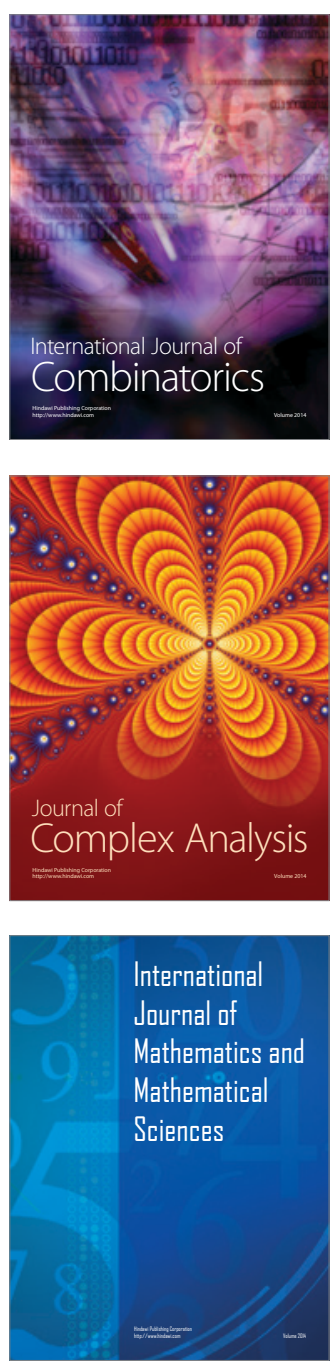
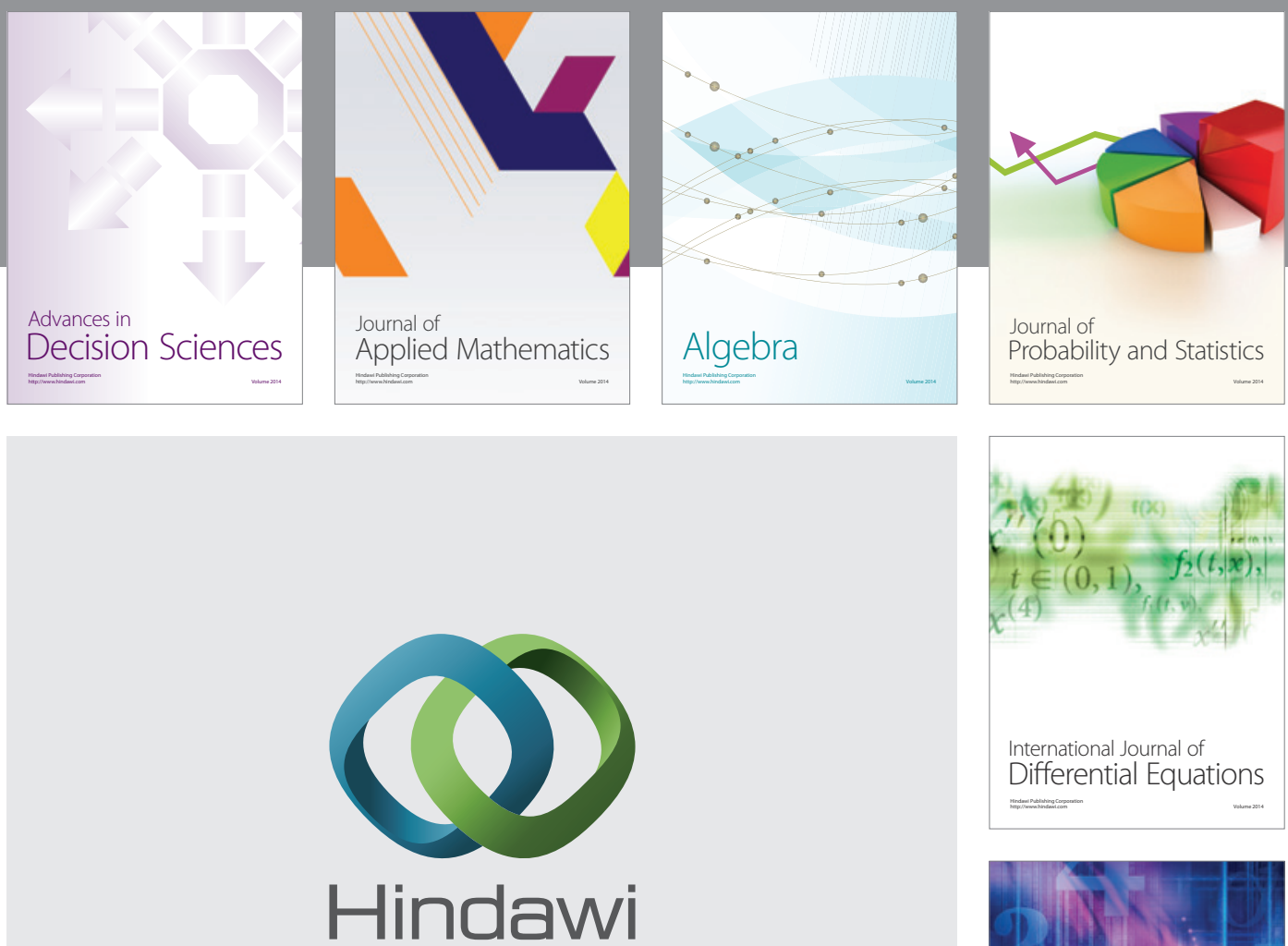

Submit your manuscripts at http://www.hindawi.com
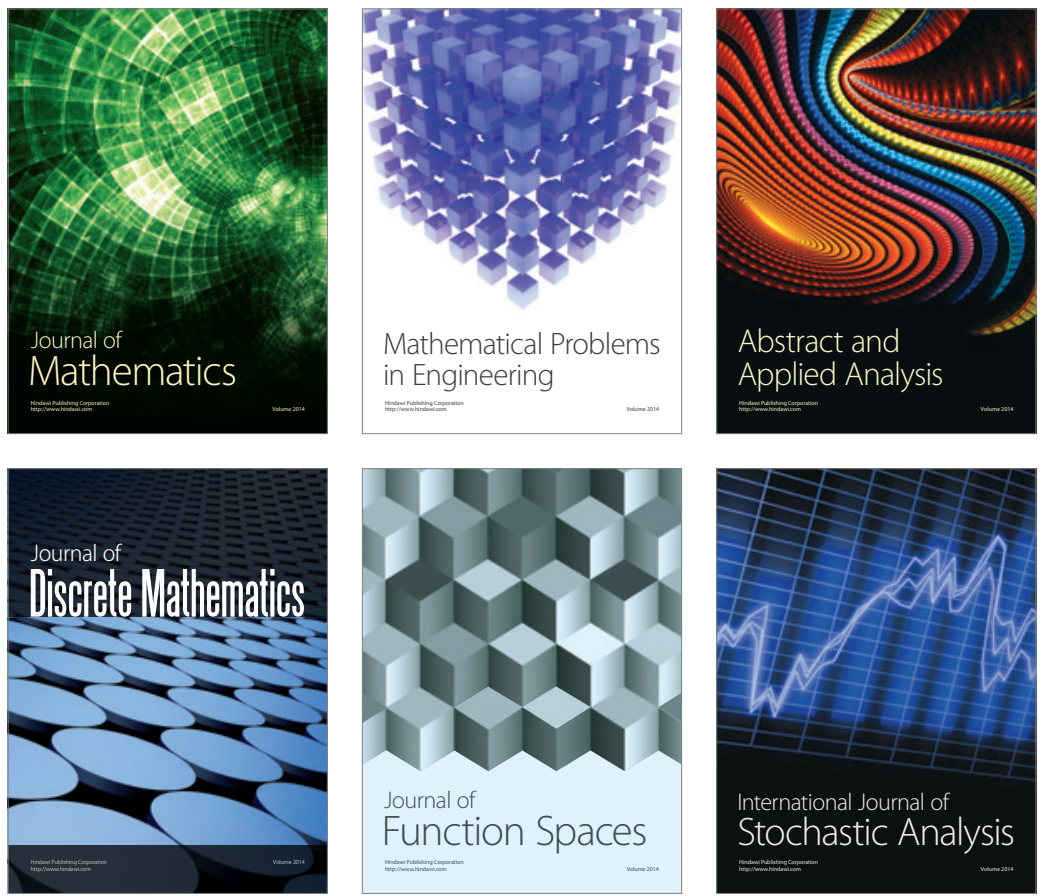

Journal of

Function Spaces

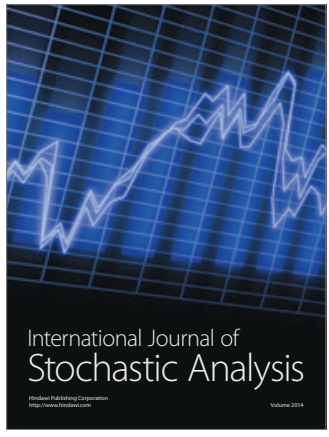

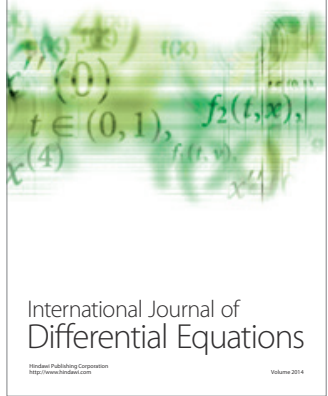
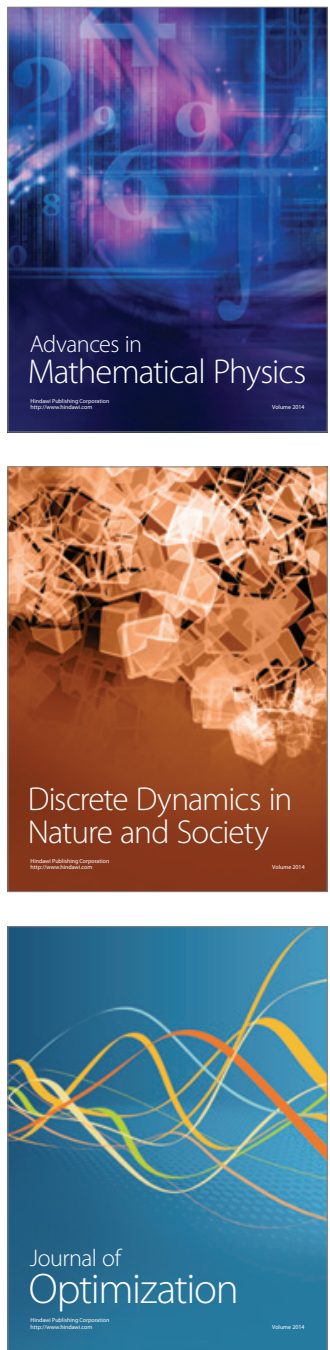